\title{
Effect of Artificial Aging on Tensile Properties and Resistance to Corrosion of 24S-T Aluminum Alloy
}

\author{
By Hugh L. Logan, Harold Hessing, and Harold E. Francis ${ }^{1}$
}

\begin{abstract}
The effect of aging commercial $24 \mathrm{~S}-\mathrm{T}$ aluminum alloy sheet, for various periods at $350^{\circ}, 375^{\circ}, 385^{\circ}$ and $400^{\circ} \mathrm{F}$, on its tensile properties and resistance to corrosion was determined. Aging for 3 hours at $385^{\circ} \mathrm{F}$ produced an increase in yield strength of about 25 percent above an initial value of about $50,000 \mathrm{lb} / \mathrm{in}^{2}$, an increase in tensile strength of about 3 percent above an initial value of $70,000 \mathrm{lb} / \mathrm{in}^{2}{ }^{2}$, and a decrease to about one-third of the initial elongation of 17 to 18 percent. Approximately the same values for these properties were obtained by aging the material for 20 hours at $350^{\circ} \mathrm{F}, 5$ hours at $375^{\circ} \mathrm{F}$, or $1 \frac{1}{2}$ hours at $400^{\circ} \mathrm{F}$. Materials aged 3 to 10 hours at $385^{\circ} \mathrm{F}$ and 6 to 12 hours at $375^{\circ} \mathrm{F}$ were generally immune to stress-corrosion cracking and were no more severely damaged in corrosive media, $\mathrm{NaCl}+\mathrm{H}_{2} \mathrm{O}_{2}$ solution or marine atmosphere, than the commercially heat-treated material exposed without artificial aging.
\end{abstract}

\section{Introduction}

Various authors have reported that the yield strength of heat-treated and strained $24 \mathrm{~S}-\mathrm{T}$ aluminum alloy sheet can be appreciably increased by aging at $350^{\circ}$ to $400^{\circ} \mathrm{F}$. However, it has also been shown that heating this alloy may increase its susceptibility to intercrystalline corrosion.

Authors $[1,2]^{2}$ who have reported that the yield strength of the heat-treated and strained alloy can be increased as much as 50 percent by aging at temperatures of $350^{\circ}$ to $400^{\circ} \mathrm{F}$, also found that the ultimate tensile strength was increased 5 to 10 percent by this same treatment. Slow cooling of the $24 \mathrm{~S}-\mathrm{T}$ aluminum alloy from the solution heat-treating temperature [3], or aging of duralumin at temperatures of $135^{\circ} \mathrm{C}$ $\left(275^{\circ} \mathrm{F}\right)$ and $200^{\circ} \mathrm{C}\left(392^{\circ} \mathrm{F}\right)$ [4], have been shown to markedly increase the susceptibility of these materials to intercrystalline corrosion. This occurs almost entirely at, or immediately adjacent to, the grain boundaries, and may be very damaging to the ultimate tensile strength and ductility (as measured by percent elongation) of the mate-

1 Formerly at the National Bureau of Standards.

2 Figures in brackets indicate the literature references at the end of this paper. rial. Furthermore, it has been shown [5] that the application of stress to a material, susceptible to intercrystalline corrosion and exposed in a corrosive medium, accelerates the corrosive attack. On the other hand, the corrosion damage to material, susceptible only to the pitting type of corrosion at the surface, is not appreciably increased by the application of stress to the material.

It was the purpose of this project, undertaken at the request of and with financial assistance from the Bureau of Aeronautics, Navy Department, to determine if it was possible to heat treat the material to take advantage of the increased yield strength without increasing the danger of intercrystalline corrosion.

\section{Materials}

Commercially heat-treated $24 \mathrm{~S}-\mathrm{T}$ aluminum alloy sheet is normally strained approximately 1 percent after solution heat treatment in order to produce a flat product. The specimens used in this investigation were prepared from two commercial $24 \mathrm{~S}-\mathrm{T}$ aluminum alloy flat sheets each $48 \mathrm{in.}$ by $144 \mathrm{in.} \mathrm{by} 0.064 \mathrm{in}$. The sheets were obtained from different sources. They will be 
referred to below as sheet $A$ and sheet $B$. The chemical compositions of the two sheets were:

\begin{tabular}{|l|r|r|}
\hline & Sheet $A$ & Sheet $B$ \\
\cline { 2 - 3 } & Percent & Percent \\
Copper & 4.5 & 4.3 \\
Magnesium & 1.5 & 1.4 \\
Manganese & 0.6 & 0.6 \\
Iron & .25 & .43 \\
Silicon & .19 & .27 \\
\end{tabular}

In addition to the above elements, both sheets contained traces of calcium, chromium, gallium, lead, nickel, silver, tin, titanium, and vanadium, as determined spectroscopically. Sheet $B$ also contained a trace of zinc.

\section{Testing Procedure}

The method of test consisted of aging the materials for various times at temperatures of $350^{\circ}, 375^{\circ}, 385^{\circ}$, and $400^{\circ} \mathrm{F}$ and subsequently determining their tensile properties as aged and after exposure in various corrosive media. The term "aging" as used hereafter will refer to artificial or elevated-temperature aging and not to the spontaneous aging that takes place at room temperature after the solution heat treatment of the $24 \mathrm{~S}$ alloy. Specimens were exposed for 24 hours, stressed in tension to three-fourths of the yield strength, in a sodium chloride-hydrogen peroxide solution ( $\mathrm{NaCl}, 57 \mathrm{~g} ; \mathrm{H}_{2} \mathrm{O}_{2}(30 \%)$ $10 \mathrm{ml} ; \mathrm{H}_{2} \mathrm{O}, 990 \mathrm{ml}$ ) [6] in the laboratory. Immersion in this solution has proved to be a satisfactory accelerated test to indicate the type of corrosion that may be expected to develop in aluminum alloys of the duralumin type in marine exposure [7]. Specimens were also exposed in the weather unstressed and stressed, as indicated above, and unstressed in tidewater, all at the Naval Air Station, Hampton Roads, Va. The changes in tensile properties, particularly the ultimate tensile strength and percentage elongation, resulting from exposure of the materials in the corroding media were taken as a measure of the corrosion damage. The type of corrosive attack was determined by metallographic examination of the exposed material.

Prior to the elevated-temperature aging treatment, most of the material was machined into standard ASTM 1/2-in. reduced-section tensile specimens. Machined tensile specimens were artificially aged in an oven that had been brought to the desired temperature before it was charged with specimens. The oven was provided with a blower for air circulation and thermostatic control to $\pm 3^{\circ} \mathrm{F}$. A period of 8 to 12 minutes was required to bring the thermostat element back to the desired temperature after placing the specimens in the oven. The aging period was measured from that time, on the assumption that the specimens, which were well separated and small in volume, reached the oven temperature along with the thermostat element.

Panels 6 in. by 14 in. for exposure (unstressed) in a marine atmosphere and for intermittent immersion in tidewater were cut from the original sheet materials, 0.064 in. thick, described above, and were aged for various periods in a tempering furnace at $385^{\circ} \pm 10^{\circ} \mathbf{F}$. At the end of all aging treatments, the specimens were quenched into tap water at room temperature.

All tensile tests were made with a hydraulic type of testing machine having a 5,000-pound load range. The cross-head speed was approximately 0.05 in. per minute. Yield strengths were obtained from load-strain diagrams drawn by a Templin type high-magnification stress-strain recorder. The values reported for the tensile properties are the averages obtained from three or more specimens.

Stress-corrosion tests were made on specimens loaded axially by means of simple lever systems. The stress-corrosion rack used in the laboratory for testing many of these specimens is shown in figure 1 .

The cells were of Pyrex glass, $60 \mathrm{~mm}$ outside diameter, fitted into grooves in the Bakelite disks at the top and bottom. Breakage of the cylinders was reduced and watertight seals were made by using rubber gaskets between the glass and Bakelite. Rubber stoppers (molded by the Rubber Section of the National Bureau of Standards) containing rectangular slots slightly smaller than the grip ends of the specimens fitted into tapered holes in the lower Bakelite disks. Use of these stoppers facilitated the insertion and removal of specimens and at the same time prevented leakage of the corroding solution.

Three specimens for each aging period, for specimens aged at $350^{\circ}, 385^{\circ}$, or $400^{\circ} \mathrm{F}$, were immersed in the solution at room temperature 


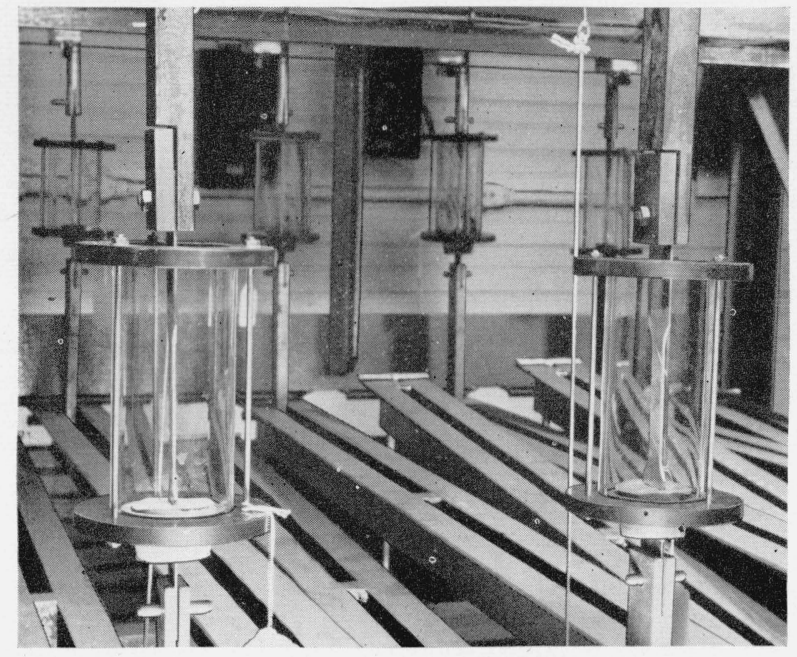

Figure 1.-Stress-corrosion rack with cells in place for tests in the $\mathrm{NaCl}+\mathrm{H}_{2} \mathrm{O}_{2}$ solution.

Specimens loaded axially by means of lever systems. Cord (right) connects knife switch and lever so that circuit to solenoid counter is opened if specimen breaks.

under stresses equal to three-fourths of the yield strength of the particular specimens under test. Room temperature was between $70^{\circ}$ and $85^{\circ} \mathrm{F}$. In order to eliminate temperature as a factor in the corrosion testing, and also to evaluate the effect of stress as a factor in corrosion damage, two sets of specimens for each aging period at $375^{\circ} \mathrm{F}$ were immersed in the $\mathrm{NaCl}+\mathrm{H}_{2} \mathrm{O}_{2}$ solution at a temperature of $95^{\circ} \pm 1^{\circ} \mathrm{F}$; one set of specimens was unstressed, the second set was stressed to three-fourths of the yield strength. The temperature $95^{\circ} \mathrm{F}$ was selected, on recommendation of the Bureau of Aeronautics, because salt-spray corrosion data are obtained at that temperature.

To supplement the laboratory stress-corrosion tests, tensile specimens aged at $375^{\circ}$ and $385^{\circ} \mathrm{F}$ were exposed in a marine atmosphere at the Naval Air Station, Hampton Roads, Va. Tensile specimens aged at $385^{\circ} \mathrm{F}$ were anodized before being exposed; specimens aged at $375^{\circ} \mathrm{F}$ were exposed with no surface treatment other than degreasing. The racks used in exposing the specimens are shown in figure 2, and fixtures for holding specimens and the specimens themselves are illustrated in figure 3. The device for measuring times to failure of the specimens is shown in figure 4 . Loads were applied by means of lever systems and weights. Specimens were loaded to produce stresses, in the reduced sections, approximately

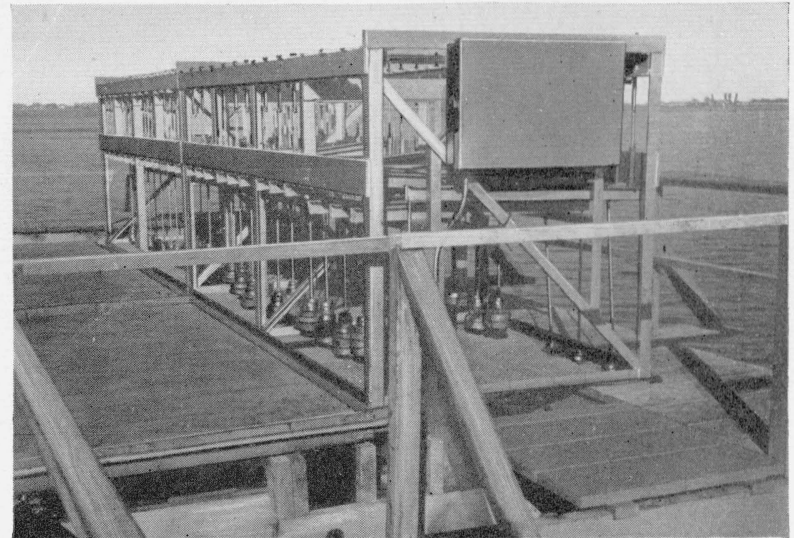

Figure 2.-Stress-corrosion racks, Naval Air Station, Hampton Roads, Va.
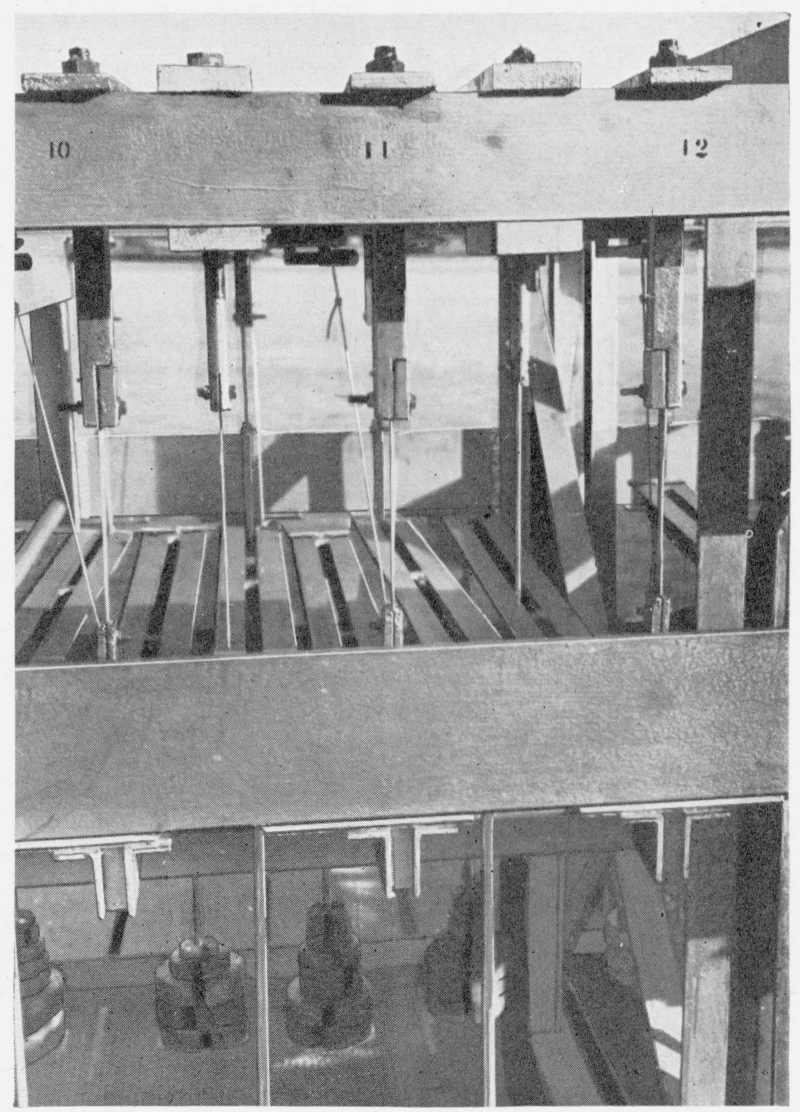

FIGURE 3.- Stressed and unstressed specimens in marineatmosphere stress-corrosion rack.

equal to three-fourths of the yield strengths, and were exposed for approximately 6 weeks. Specimens aged at $375^{\circ} \mathrm{F}$ were also exposed to the marine atmosphere unstressed, but with conditions otherwise identical with those for the stressed specimens. 


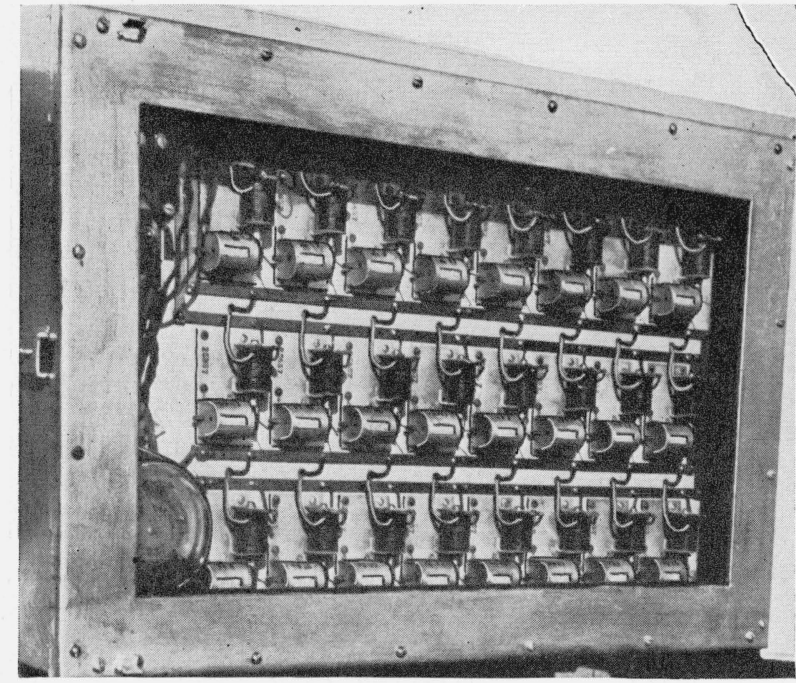

FIGURE 4.-Electric solenoid counters and time switch (lower left).

Counters are connected in series with knife switches located above individual specimens (see fig. 3). Counters are actuated once each $6 \mathrm{~min}$ (by electric time switch) until knife switch is opened upon failure of specimen, i. e., time to failure of specimen is recorded in 0.1 -hr unit.

The panels for unstressed weather exposure and tidewater tests at Hampton Roads were heat treated and were then cut into two parts, each 3 in. by $14 \mathrm{in.}$ One part of each panel was exposed to intermittent immersion in tidewater for 14 days; the other part was exposed to marine weather for 1 year. The test sites and methods of mounting specimens have been described in a report of another investigation [8]. After exposure, the panels were returned to the Bureau, and their tensile properties determined.

The type and extent of corrosive attack on the materials were determined, after their removal from the corrosive media, by metallographic examination of one or more coupons from each set of the corroded specimens.

\section{Results}

The tensile properties of the commercially heat-treated material, without elevated-temperature aging, as determined from specimens taken parallel and transversely to the direction of rolling, and hereafter designated parallel and transverse respectively, were:

\begin{tabular}{|c|c|c|c|c|c|c|}
\hline \multirow{2}{*}{ Material } & \multicolumn{2}{|c|}{ Yield strength 1} & \multicolumn{2}{|c|}{$\begin{array}{l}\text { Ultimate tensile } \\
\text { strength }\end{array}$} & \multicolumn{2}{|c|}{$\begin{array}{c}\text { Elongation in } \\
2 \text { in. }\end{array}$} \\
\hline & Parallel & $\begin{array}{l}\text { Trans- } \\
\text { verse }\end{array}$ & Parallel & $\begin{array}{l}\text { Trans- } \\
\text { verse }\end{array}$ & Parallel & $\begin{array}{c}\text { Trans- } \\
\text { verse }\end{array}$ \\
\hline $\begin{array}{l}A \\
B \\
B\end{array}$ & $\begin{array}{l}l b / \text { in. }^{2} \\
54,300 \\
50,500\end{array}$ & $\begin{array}{l}\text { lb/in. }{ }^{2} \\
47,600 \\
44,400\end{array}$ & $\begin{array}{l}l b / \text { in }^{2} \\
71,700 \\
67,300\end{array}$ & $\begin{array}{l}\text { lb/in. }{ }^{2} \\
70,700 \\
67,200\end{array}$ & $\begin{array}{r}\text { Per- } \\
\text { cent } \\
17 \\
18\end{array}$ & $\begin{array}{l}\text { Per- } \\
\text { cent } \\
18 \\
181 / 2\end{array}$ \\
\hline
\end{tabular}

10.2 percent offset from the modulus line.

Specimens taken parallel to the direction of rolling were aged at $350^{\circ} \mathrm{F}$ for periods of time from 2 to 24 hours; at $375^{\circ} \mathrm{F}$ for periods of 2 to 12 hours; at $385^{\circ} \mathrm{F}$ for 8 minutes to 12 hours; and at $400^{\circ} \mathrm{F}$ for 1 to 6 hours. Specimens taken transversely to the direction of rolling were aged for periods of time from 1 to 8 hours at $385^{\circ} \mathrm{F}$. The results of the aging treatments and corrosion tests are given in tables 1 to 6 and are shown graphically in figures 5 to 20 , inclusive. Standard deviations for yield strengths, etc., given in the tables were computed from averages of ranges [9]. 
TABLE 1.-Effect of aging period at $385^{\circ} \mathrm{F}$ on tensile properties and resistance to corrosion of 0.064 -in.-gage commercial 24S-T aluminum-alloy sheet, lot $A$

\begin{tabular}{|c|c|c|c|c|c|c|c|c|c|c|}
\hline \multirow{2}{*}{$\begin{array}{l}\text { Aging time } \\
\text { at } 385^{\circ} \mathrm{F}\end{array}$} & \multicolumn{3}{|c|}{$\begin{array}{l}\text { Tensile properties of aged } \\
\text { material }\end{array}$} & \multirow{2}{*}{$\begin{array}{c}\text { Stress in } \\
\text { corroding } \\
\text { medium }\end{array}$} & \multicolumn{3}{|c|}{$\begin{array}{l}\text { Tensile properties after } 24 \mathrm{hr} \text {. } \\
\text { under stress in } \mathrm{NaCl}+\mathrm{H}_{2} \mathrm{O}_{2} \\
\text { solution }\end{array}$} & \multicolumn{3}{|c|}{$\begin{array}{l}\text { Tensile properties after } 6 \text { weeks } \\
\text { under stress in marine atmos- } \\
\text { phere }{ }^{2}\end{array}$} \\
\hline & $\begin{array}{l}\text { Yield } \\
\text { strength } 1\end{array}$ & $\begin{array}{l}\text { Ultimate } \\
\text { tensile } \\
\text { strength }\end{array}$ & $\begin{array}{c}\text { Elonga- } \\
\text { tion in } \\
2 \text { in. }\end{array}$ & & $\begin{array}{l}\text { Yield } \\
\text { strength }\end{array}$ & $\begin{array}{l}\text { Ultimate } \\
\text { tensile } \\
\text { strength }\end{array}$ & $\begin{array}{l}\text { Elonga- } \\
\text { tion in } \\
2 \text { in. }\end{array}$ & $\begin{array}{l}\text { Yield } \\
\text { strength }\end{array}$ & $\begin{array}{c}\text { Ultimate } \\
\text { tensile } \\
\text { strength }\end{array}$ & $\begin{array}{l}\text { Elonga- } \\
\text { tion in } \\
2 \text { in. }\end{array}$ \\
\hline \multicolumn{11}{|c|}{ PARALLEL SPECIMENS } \\
\hline Hours & $l b /$ in. $^{2}$ & $l b / i n .^{2}$ & Percent & $l b / i n .^{2}$ & $l b /$ in $^{2}$ & $l b /$ in $^{2}$ & Percent & lb/in.2 & $l b / i n .^{2}$ & Percent \\
\hline 0 & 54,300 & 71,700 & 17 & 40,500 & 46,800 & 53,600 & 3 & 52,800 & 70,700 & 12 \\
\hline 2/15 & 51,300 & 70,400 & $171 / 2$ & 38,500 & 42,200 & 45,700 & $21 / 2$ & - - - & - n- & -..... \\
\hline $1 / 4$ & 49,100 & 70,200 & 19 & 36,800 & 42,300 & 47,300 & 3 & 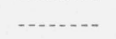 & . & - n- \\
\hline $1 / 2$ & 55,700 & 70,900 & 17 & 41,700 & 44,900 & 48,300 & 3 & ........ & ......... & - n-...- \\
\hline 1 & 62,000 & 72,200 & $121 / 2$ & 47,600 & 56,100 & 61,800 & 4 & 63,000 & 70,200 & $81 / 2$ \\
\hline 2 & 66,700 & 73,800 & $81 / 2$ & 50,700 & 59,500 & 63,800 & $31 / 2$ & 67,200 & 74,200 & 5 \\
\hline $21 / 2$ & 67,400 & 74,100 & $81 / 2$ & -...... & - & - & - & -..-1 & - & -....... \\
\hline 3 & 68,600 & 73,400 & 7 & 51,200 & 61,400 & 66,000 & $41 / 2$ & 65,600 & 72,500 & 6 \\
\hline 4 & 67,000 & 72,700 & $71 / 2$ & 50,100 & 61,100 & 65,000 & 4 & 66,700 & 72,900 & 6 \\
\hline 5 & 67,000 & 73,300 & $61 / 2$ & 50,700 & 62,000 & 65,800 & 4 & -.... & - & -....... \\
\hline 6 & 65,500 & 72,200 & 6 & 48,800 & 57,600 & 62,500 & $31 / 2$ & 62,000 & 70,700 & 7 \\
\hline 7 & 64,500 & 71,000 & $61 / 2$ & 48,400 & 59,100 & 64,800 & 4 & -..... & -........ & -... \\
\hline 8 & 63,800 & 71,400 & 7 & 48,000 & 57,500 & 63,800 & 3 & 62,600 & 71,200 & 7 \\
\hline 10 & 63,100 & 70,900 & $61 / 2$ & 47,900 & 57,400 & 63,800 & $31 / 2$ & - & -....... & - \\
\hline 12 & 64,300 & 71,200 & $61 / 2$ & 47,900 & 58,200 & 63,900 & $31 / 2$ & 64,700 & 70,800 & $61 / 2$ \\
\hline $\begin{array}{l}\text { Stand ar d } \\
\text { deviation. }{ }^{3}\end{array}$ & 1,130 & 725 & 0.70 & - & 955 & 1,425 & 0.50 & 1,350 & 1,140 & 0.80 \\
\hline \multicolumn{11}{|c|}{ TRANSVERSE SPECIMENS } \\
\hline 0 & 47,600 & 70,700 & 18 & 35,700 & 42,800 & 47,800 & 2 & - & 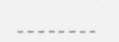 & - \\
\hline 1 & 57,800 & 72,500 & 13 & 45,300 & 51,700 & 55,300 & $21 / 2$ & - & - & - \\
\hline 2 & 63,000 & 71,800 & 8 & 47,300 & 56,800 & 62,500 & $31 / 2$ & - & - & -... \\
\hline $21 / 2$ & 63,400 & 70,100 & 6 & 47,600 & 58,200 & 62,100 & $21 / 2$ & - & - & - \\
\hline 3 & 65,900 & 72,300 & $61 / 2$ & 49,300 & 59,500 & 64,100 & $3 \frac{1}{2}$ & - n- & - n-n & - n \\
\hline 4 & 65,700 & 71,900 & $61 / 2$ & 49,300 & 57,000 & 60,000 & $21 / 2$ & . & - & - \\
\hline 5 & 66,100 & 72,400 & 6 & 49,600 & 58,700 & 61,800 & $21 / 2$ & - & - & - \\
\hline 6 & 64,700 & 70,900 & 6 & 48,200 & 58,100 & 61,000 & 2 & - & . & -..... \\
\hline 8 & 62,800 & 70,300 & $61 / 2$ & 47,200 & - . & 60,800 & $21 / 2$ & - & . & - \\
\hline $\begin{array}{l}\text { Standard } \\
\text { deviation. }{ }^{3}\end{array}$ & 945 & 415 & 0.60 & 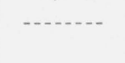 & 1,090 & 1,600 & 0.55 & - n & - n & - n \\
\hline
\end{tabular}

${ }^{1} 0.2 \%$ offset from modulus line.

2 Material inserted in rack $12 / 23 / 44$, removed 2/2/45. These specimens anodized before exposure.

${ }^{3}$ The standard deviation was determined by dividing the average range by 1.693; see Simon, "Engineer's manual of statistical methods", p. 138. Range equals maximum value minus minimum value. 
TABLE 2.-Effect of aging period at $385^{\circ} \mathrm{F}$ on tensile properties and resistance to corrosion of 0.064-in.-gage 24S-T commercial aluminum alloy, lot $B$

\begin{tabular}{|c|c|c|c|c|c|c|c|c|c|c|}
\hline \multirow{2}{*}{$\begin{array}{l}\text { Aging period } \\
\text { at } 385^{\circ} \mathrm{F}\end{array}$} & \multicolumn{3}{|c|}{ Tensile properties of aged material } & \multirow{2}{*}{$\begin{array}{l}\text { Stress in } \\
\text { corroding } \\
\text { medium }\end{array}$} & \multicolumn{3}{|c|}{$\begin{array}{l}\text { Tensile properties after } 24 \mathrm{hr} \text { in } \\
\mathrm{NaCl}+\mathrm{H}_{2} \mathrm{O}_{2} \text { solution under stress }\end{array}$} & \multicolumn{3}{|c|}{$\begin{array}{l}\text { Tensile properties after } 6 \text { weeks } \\
\text { under stress in marine atmosphere }\end{array}$} \\
\hline & $\begin{array}{l}\text { Yield } \\
\text { strength }\end{array}$ & $\begin{array}{l}\text { Ultimate } \\
\text { tensile } \\
\text { strength }\end{array}$ & $\begin{array}{l}\text { Elonga- } \\
\text { tion in } \\
2 \text { in. }\end{array}$ & & $\begin{array}{l}\text { Yield } \\
\text { strength }\end{array}$ & $\begin{array}{l}\text { Ultimate } \\
\text { tensile } \\
\text { strength }\end{array}$ & $\begin{array}{l}\text { Elonga- } \\
\text { tion in } \\
2 \text { in. }\end{array}$ & $\begin{array}{l}\text { Yield } \\
\text { strength }\end{array}$ & $\begin{array}{l}\text { Ultimate } \\
\text { tensile } \\
\text { strength }\end{array}$ & $\begin{array}{l}\text { Elonga- } \\
\text { tion in } \\
2 \text { in. }\end{array}$ \\
\hline \multicolumn{11}{|c|}{ PARALLEL SPECIMENS } \\
\hline Hours & $l b /$ in $^{2}$ & $l b, i n .^{2}$ & Percent & lb/in.2 ${ }^{2}$ & lb/in..$^{2}$ & lb/in. ${ }^{2}$ & Percent & $\not b /$ in $^{2}$ & lb/in..$^{2}$ & Percent \\
\hline 0 & 50,500 & 67,300 & 18 & 38,300 & 47,100 & 58,800 & 7 & 49,500 & 65,700 & 9 \\
\hline 215 & 48,200 & 66,700 & 18 & 36,900 & 41,700 & 46,900 & $21 / 2$ & - n. & -....... & - n.... \\
\hline $1 / 4$ & 47,600 & 66,200 & 19 & 36,800 & 41,100 & 47,600 & 3 & - n & - n. & - n.... \\
\hline $1 / 2$ & 50,500 & 68,200 & 18 & 37,000 & 43,700 & 49,500 & 3 & -....... & - n. & - n.... \\
\hline 1 & 51,400 & 66,100 & 16 & 40,300 & 45,200 & 50,400 & 3 & 56,500 & 61,900 & $31 / 2$ \\
\hline $11 / 2$ & 57,600 & 68,100 & 11 & 44,000 & 51,100 & 57,600 & $31 / 2$ & - & - & -..... \\
\hline 2 & 62,000 & 68,900 & 8 & 46,900 & 55,100 & 59,900 & 4 & 63,300 & 68,400 & $51 / 2$ \\
\hline $21 / 2$ & 62,100 & 69,000 & $71 / 2$ & - . & -... & -....... & -..... & - & - & - . \\
\hline 3 & 62,500 & 68,400 & 7 & 47,000 & 55,500 & 60,500 & $31 / 2$ & 63,500 & 68,300 & 5 \\
\hline 4 & 61,900 & 67,900 & 7 & 46,300 & 55,900 & 60,600 & 4 & 63,400 & 69,600 & $51 / 2$ \\
\hline 5 & 59,000 & 67,500 & $61 / 2$ & 44,500 & 53,700 & 58,900 & 3 & - n & - & ..... \\
\hline 6 & 61,700 & 67,600 & $61 / 2$ & 46,300 & 56,100 & 60,400 & 3 & 61,800 & 67,600 & $51 / 2$ \\
\hline 7 & 58,800 & 66,600 & $61 / 2$ & 44,100 & 53,500 & 58,500 & $21 / 2$ & - & - & -... \\
\hline 8 & 60,100 & 66,600 & 6 & 45,800 & 54,700 & 58,600 & $21 / 2$ & 59,900 & 66,900 & 6 \\
\hline 10 & 59,900 & 67,100 & 6 & 45,000 & 53,800 & 59,000 & 3 & (n) & (n) & -...- \\
\hline 12 & 57,000 & 65,100 & $61 / 2$ & 43,900 & 50,700 & 55,100 & $21 / 2$ & 58,900 & 66,700 & $51 / 2$ \\
\hline $\begin{array}{l}\text { Standard } \\
\text { deviation. }\end{array}$ & 910 & 605 & 0.4 & - n..... & 690 & 960 & 0.45 & 515 & 1,260 & 1.4 \\
\hline \multicolumn{11}{|c|}{ TRANSVERSE SPECIMENS } \\
\hline 0 & 44,400 & 67,200 & $181 / 2$ & 33,700 & 43,400 & 59,800 & 6 & -. & $\ldots$ & - . \\
\hline 1 & 50,100 & 67,600 & 15 & 38,000 & 44,900 & 51,600 & 3 & - & - & -...... \\
\hline 2 & 59,900 & 68,100 & $71 / 2$ & 45,400 & 53,800 & 60,000 & 4 & - & ....... & - \\
\hline $21 / 2$ & 56,300 & 65,700 & $61 / 2$ & 42,500 & 52,900 & 59,400 & $31 / 2$ & - & - . & -...... \\
\hline 3 & 62,900 & 70,200 & $61 / 2$ & 47,600 & 54,500 & 60,000 & 4 & ........ & -........ & (n) \\
\hline 4 & 61,400 & 68,000 & $61 / 2$ & 46,600 & 54,900 & 60,400 & $31 / 2$ & - & - & - \\
\hline 5 & 61,100 & 67,800 & $61 / 2$ & 46,300 & 55,300 & 60,600 & $31 / 2$ & - & ....... & - \\
\hline 6 & 59,300 & 66,800 & 6 & 44,800 & 53,300 & 59,100 & 3 & - & - & -...... \\
\hline 8 & 61,000 & 68,200 & 6 & 45,700 & - & 59,600 & $21 / 2$ & - & - & -..... \\
\hline $\begin{array}{l}\text { Standard } \\
\text { deviation. }\end{array}$ & 610 & 505 & 1.0 & -...... & 575 & 860 & 0.6 & - & - & ...... \\
\hline
\end{tabular}


TABLE 3.-Tensile properties of material before and after exposure in tidewater for 2 weeks or in a marine atmosphere for 1 year

\begin{tabular}{|c|c|c|c|c|c|c|c|c|c|}
\hline \multirow{2}{*}{$\begin{array}{l}\text { Aging period } \\
\text { at } 385^{\circ} \mathrm{F} \text {. }\end{array}$} & \multicolumn{3}{|c|}{$\begin{array}{l}\text { Properties of aged material } \\
\text { (unexposed) }\end{array}$} & \multicolumn{3}{|c|}{$\begin{array}{l}\text { Properties of material after } 14 \text { days' } \\
\text { exposure in tidewater }\end{array}$} & \multicolumn{3}{|c|}{$\begin{array}{l}\text { Properties of material after } 1 \text { year's } \\
\text { exposure in marine atmosphere }\end{array}$} \\
\hline & $\begin{array}{l}\text { Yield } \\
\text { strength }\end{array}$ & $\begin{array}{l}\text { Ultimate } \\
\text { tensile } \\
\text { strength }\end{array}$ & $\begin{array}{l}\text { Elongation } \\
\text { in } 2 \text { in. }\end{array}$ & $\begin{array}{l}\text { Yield } \\
\text { strength }\end{array}$ & $\begin{array}{l}\text { Ultimate } \\
\text { tensile } \\
\text { strength }\end{array}$ & $\begin{array}{l}\text { Elongation } \\
\text { in } 2 \text { in. }\end{array}$ & $\begin{array}{l}\text { Yield } \\
\text { strength }\end{array}$ & $\begin{array}{c}\text { Ultimate } \\
\text { tensile } \\
\text { strength }\end{array}$ & $\begin{array}{l}\text { Elongation } \\
\text { in } 2 \text { in. }\end{array}$ \\
\hline \multicolumn{10}{|c|}{$A$ MATERIAL } \\
\hline Hours & $l b /$ in $^{2}$ & $l b /$ in. $^{2}$ & Percent & $l b / i n .^{2}$ & $l b /$ in. $^{2}$ & Percent & $l b /$ in..$^{2}$ & $l b /$ in..$^{2}$ & Percent \\
\hline 0 & 54,300 & 71,700 & 17 & 54,200 & 70,800 & $151 / 2$ & 54,600 & 70,700 & $16 \frac{1}{2}$ \\
\hline 1 & 62,000 & 72,200 & $12 \frac{1}{2}$ & 54,900 & 68,900 & $101 / 2$ & 44,500 & 49,500 & 5 \\
\hline 2 & 66,700 & 73,800 & $81 / 2$ & 61,500 & 72,900 & 10 & 60,900 & 70,400 & 9 \\
\hline $21 / 2$ & 67,400 & 74,100 & $81 / 2$ & 65,100 & 74,200 & $91 / 2$ & 63,200 & 71,700 & 8 \\
\hline 3 & 68,600 & 73,400 & 7 & 66,400 & 73,700 & $81 / 2$ & 65,600 & 73,200 & 8 \\
\hline 4 & 67,000 & 72,700 & $71 / 2$ & 67,300 & 73,800 & 7 & 66,100 & 73,300 & 7 \\
\hline 5 & 67,000 & 73,300 & $61 / 2$ & 68,200 & 73,400 & 6 & 67,000 & 72,700 & 6 \\
\hline 6 & 65,500 & 72,200 & 6 & 67,800 & 72,800 & $41 / 2$ & 66,900 & 72,500 & $51 / 2$ \\
\hline 8 & 63,800 & 71,400 & 7 & 65,100 & 70,900 & $51 / 2$ & 63,500 & 70,500 & 6 \\
\hline $\begin{array}{l}\text { Standard de- } \\
\text { viation. }\end{array}$ & 1,050 & 750 & 0.55 & 335 & 530 & 0.85 & 1,070 & 1,550 & 0.70 \\
\hline \multicolumn{10}{|c|}{$B$ MATERIAL } \\
\hline 0 & 50,500 & 67,300 & 18 & 51,100 & 67,700 & 16 & 50,700 & 67,500 & 15 \\
\hline 1 & 51,400 & 66,100 & 16 & 51,400 & 64,900 & $81 / 2$ & 50,800 & 63,600 & 10 \\
\hline 2 & 62,000 & 68,900 & 8 & 55,500 & 65,700 & $61 / 2$ & 54,700 & 66,100 & $81 / 2$ \\
\hline $21 / 2$ & 62,100 & 69,000 & $71 / 2$ & 58,500 & 64,800 & 3 & 55,700 & 66,100 & 7 \\
\hline 3 & 62,500 & 68,400 & 7 & 60,100 & 67,100 & 5 & 58,700 & 66,800 & 6 \\
\hline 4 & 61,900 & 67,900 & 7 & 62,400 & 66,900 & $41 / 2$ & 61,700 & 68,300 & 6 \\
\hline 5 & 61,100 & 68,200 & 7 & 62,900 & 66,700 & $41 / 2$ & 62,200 & 68,100 & $51 / 2$ \\
\hline 6 & 61,700 & 67,600 & $61 / 2$ & 61,000 & 65,200 & 3 & 61,000 & 67,600 & 4 \\
\hline 8 & 60,100 & 66,600 & 6 & 60,600 & 66,000 & 4 & 60,400 & 66,700 & $51 / 2$ \\
\hline $\begin{array}{l}\text { Standard de- } \\
\text { viation. }\end{array}$ & 490 & 520 & 0.40 & 610 & 1,085 & 0.95 & 790 & 270 & 0.80 \\
\hline
\end{tabular}


TABLE 4.-Effect of aging period at $375^{\circ} \mathrm{F}$ on parallel tensile properties and resistance to corrosion of $0.064-i n .-$ gage commercial $24 S-T$ aluminum alloy

\begin{tabular}{|c|c|c|c|c|c|c|c|c|c|c|}
\hline \multirow{2}{*}{$\begin{array}{l}\text { Aging period } \\
\text { at } 375^{\circ} \mathrm{F}\end{array}$} & \multicolumn{3}{|c|}{ Tensile preperties of aged material } & \multirow{2}{*}{$\begin{array}{l}\text { Stress in } \\
\text { corroding } \\
\text { medium }\end{array}$} & \multicolumn{3}{|c|}{$\begin{array}{l}\text { Tensile properties after } 24 \mathrm{hr} \text { in } \\
\mathrm{NaCl}+\mathrm{H}_{2} \mathrm{O}_{2} \text { solution }\end{array}$} & \multicolumn{3}{|c|}{$\begin{array}{l}\text { Tensile properties after } 6 \text { weeks in } \\
\text { marine atmosphere }{ }^{1}\end{array}$} \\
\hline & $\begin{array}{l}\text { Yield } \\
\text { strength }\end{array}$ & $\begin{array}{l}\text { Ultimate } \\
\text { tensile } \\
\text { strength }\end{array}$ & $\begin{array}{l}\text { Elonga- } \\
\text { tion in } \\
2 \text { in. }\end{array}$ & & $\begin{array}{l}\text { Yield } \\
\text { strength }\end{array}$ & $\begin{array}{l}\text { Ultimate } \\
\text { tensile } \\
\text { strength }\end{array}$ & $\begin{array}{l}\text { Elonga- } \\
\text { tion in } \\
2 \text { in. }\end{array}$ & $\begin{array}{l}\text { Yield } \\
\text { strength }\end{array}$ & $\begin{array}{l}\text { Ultimate } \\
\text { tensile } \\
\text { strength }\end{array}$ & $\begin{array}{l}\text { Elonga- } \\
\text { tion in } \\
2 \text { in. }\end{array}$ \\
\hline \multicolumn{11}{|c|}{ A MATERIAL } \\
\hline Hours & $l b / i n .{ }^{2}$ & $l b / i n .^{2}$ & Percent & $l b /$ in. $^{2}$ & lb/in. ${ }^{2}$ & $l t / i n . .^{2}$ & Percent & lb/in..$^{2}$ & $l b /$ in. $^{2}$ & Percent \\
\hline 0 & 54,300 & 71,700 & 17 & 40,500 & 47,600 & 55,300 & 4 & 54,700 & 71,900 & \\
\hline 0 & 54,300 & 71,700 & 17 & 0 & 50,900 & 65,800 & $101 / 2$ & 54,000 & 71,600 & $151 / 2$ \\
\hline 2 & 62,800 & 75,000 & $151 / 2$ & 51,300 & 56,000 & 60,100 & 4 & - nes.... & - nen & $-\ldots$ \\
\hline 2 & 62,800 & 75,000 & $15 \frac{1}{2}$ & 0 & 56,000 & 64,300 & $71 / 2$ & .......... & .......... & \\
\hline 3 & 65,100 & 74,300 & 11 & -....- & -...-... & -..--.-- & $-\ldots-$ & -...-..-- & -.......- & -...- \\
\hline 4 & 69,300 & 74,200 & $61 / 2$ & 52,000 & 63,000 & 68,600 & $5 \frac{1}{2}$ & 67,400 & 72,700 & 6 \\
\hline 4 & 69,300 & 74,200 & $61 / 2$ & 0 & 64,600 & 69,700 & 7 & 69,000 & 74,400 & $51 / 2$ \\
\hline 5 & 69,500 & 73,800 & $71 / 2$ & & ......... & ......... & ....... & ......... & ......... & ........ \\
\hline 6 & 67,600 & 73,200 & $71 / 2$ & 48 to 50,000 & 62,800 & 68,200 & $51 / 2$ & 66,000 & 72,000 & 6 \\
\hline 6 & 67,600 & 73,200 & $71 / 2$ & 0 & 60,600 & 66,700 & 5 & 64,300 & 71,700 & 6 \\
\hline 7 & 67,700 & 73,600 & 7 & & & & & & & \\
\hline 8 & 67,600 & 73,400 & 6 & 50,800 & 62,100 & 67,700 & $51 / 2$ & 66,700 & 72,200 & 6 \\
\hline 8 & 67,600 & 73,400 & 6 & 0 & 62,300 & 67,500 & 5 & 66,200 & 72,000 & 5 \\
\hline 10 & 64,500 & 71,600 & $61 / 2$ & ....... & ........ & ......... & ........ & ......... & ......... & ....... \\
\hline 12 & 64,090 & 71,300 & $61 / 2$ & 47,900 & 59,100 & 65.900 & $51 / 2$ & 61,500 & 69,900 & $51 / 2$ \\
\hline 12 & 64,000 & 71,300 & $61 \frac{1}{2}$ & 0 & 59,800 & 65,900 & 5 & 62,900 & 70,700 & 6 \\
\hline \multirow{2}{*}{$\begin{array}{l}\text { Standard } \\
\text { deviation }\end{array}$} & 560 & 495 & 0.40 & f Stressed & 1,395 & 1,715 & 1.0 & 1,275 & 970 & 1.1 \\
\hline & & & & Unstressed & 1,165 & 825 & 1.0 & 1,735 & 545 & 0.80 \\
\hline \multicolumn{11}{|c|}{$B$ MATERIAL } \\
\hline 0 & 50,500 & 67,300 & 18 & 37,900 & 43,600 & 55,000 & 6 & 49,200 & 61,300 & $81 / 2$ \\
\hline 0 & 50,500 & 67,300 & 18 & 0 & 46,300 & 61,700 & $91 / 2$ & 48,400 & 66,700 & 15 \\
\hline 2 & 56,200 & 67,900 & 13 & 42,150 & 47,800 & 55,800 & 5 & .......... & -........ & -........ \\
\hline 2 & 56,200 & 67,900 & 13 & 0 & 46,800 & 58,200 & 8 & -....... & - & -...... \\
\hline 3 & 59,200 & 68,800 & $101 / 2$ & & -........ & -........ & - & -....... & - n- & - n \\
\hline 4 & 63,200 & 69,600 & $71 / 2$ & 47,400 & 56,100 & 63,200 & $51 / 2$ & 62,500 & 67,100 & 4 \\
\hline 4 & 63,200 & 69,600 & $71 / 2$ & 0 & 55,700 & 63,300 & 6 & 61,700 & 67,300 & $41 / 2$ \\
\hline 5 & 63,300 & 68,900 & $71 / 2$ & & - n........ & -......... & - & -......... & -.......... & -.... \\
\hline 6 & 62,900 & 69,400 & $61 / 2$ & 47,200 & 57,700 & 62,900 & 4 & 60,500 & 67,000 & 4 \\
\hline 6 & 62,900 & 69,400 & $61 / 2$ & 0 & 57,300 & 63,200 & 5 & 62,000 & 68,400 & 5 \\
\hline 8 & 60,300 & 67,400 & 6 & 45,300 & 56,800 & 61,800 & $31 / 2$ & 59,600 & 66,500 & 4 \\
\hline 8 & 60,300 & 67,400 & 6 & 0 & 56,600 & 61,500 & 4 & 59,900 & 66,000 . & 4 \\
\hline 10 & 62,100 & 68,300 & $61 / 2$ & & - & -.......... & ....... & - & -..-..... & .... \\
\hline 12 & 60,800 & 67,500 & 6 & 45,500 & 56,200 & 61,600 & $41 / 2$ & 61,300 & 66,300 & 5 \\
\hline 12 & 60,800 & 67,500 & 6 & 0 & 55,800 & 61,600 & $41 / 2$ & 59,600 & 65,000 & 4 \\
\hline \multirow{2}{*}{$\begin{array}{l}\text { Standard } \\
\text { deviation }\end{array}$} & 620 & $39 j$ & 0.60 & Stressed & 885 & 650 & 0.60 & 1,040 & 1,030 & 1.0 \\
\hline & & & & Unstressed & 660 & 550 & 1.2 & 1,430 & 1,005 & 0.40 \\
\hline
\end{tabular}

${ }_{1}$ Material inserted in rack $3 / 17 / 45$, removed $4 / 28 / 45$. 
TABLE 5.-Effect of aging period at $350^{\circ} \mathrm{F}$ on parallel properties and resistance to corrosion of 0.064 -in.-gage commercial 24S-T aluminum alloy

\begin{tabular}{|c|c|c|c|c|c|c|c|}
\hline \multirow{2}{*}{$\begin{array}{l}\text { Aging period } \\
\quad \text { at } 350^{\circ} \mathrm{F}\end{array}$} & \multicolumn{3}{|c|}{ Tensile properties of aged material } & \multirow{2}{*}{$\begin{array}{l}\text { Stress in } \\
\text { corroding } \\
\text { medium }\end{array}$} & \multicolumn{3}{|c|}{$\begin{array}{c}\text { Tensile properties after } 24 \mathrm{hr} \text { in } \\
\mathrm{NaCl}+\mathrm{H}_{2} \mathrm{O}_{2} \text { solution }\end{array}$} \\
\hline & $\begin{array}{l}\text { Yield } \\
\text { strength }\end{array}$ & $\begin{array}{l}\text { Ultimate } \\
\text { tensile } \\
\text { strength }\end{array}$ & $\begin{array}{c}\text { Elonga- } \\
\text { tion in } 2 \text { in. }\end{array}$ & & $\begin{array}{l}\text { Yield } \\
\text { strength }\end{array}$ & $\begin{array}{l}\text { Ultimate } \\
\text { tensile } \\
\text { strength }\end{array}$ & $\begin{array}{l}\text { Elonga- } \\
\text { tion in } 2 \text { in. }\end{array}$ \\
\hline \multicolumn{8}{|c|}{ A MATERIAL } \\
\hline Hours & $l b /$ in $^{2}{ }^{2}$ & $l b / i n .^{2}$ & Percent & $l b /$ in. $^{2}$ & $l b / i n .^{2}$ & $l b /$ in. $^{2}$ & Percent \\
\hline$\theta$ & 54,300 & 71,700 & 17 & 40,500 & 46,800 & 53,600 & 3 \\
\hline 2 & 55,600 & 72,000 & 17 & 41,700 & $<45,300$ & $1<48,300$ & $21 / 2$ \\
\hline 4 & 57,200 & 72,500 & $171 / 2$ & 42,900 & 45,700 & 49,200 & 3 \\
\hline 8 & 66,800 & 74,700 & $91 / 2$ & 50,100 & 56,100 & 62,000 & 3 \\
\hline 16 & 69,600 & 74,000 & $61 / 2$ & 52,200 & 61,400 & 65,800 & 3 \\
\hline 24 & 69,600 & 73,900 & 6 & 52,000 & 61,000 & 65,600 & 3 \\
\hline $\begin{array}{l}\text { Standard de- } \\
\text { viation. }\end{array}$ & 1,215 & 400 & 0.6 & (n....... & 1,160 & 2,620 & 0.5 \\
\hline \multicolumn{8}{|c|}{$B$ MATERIAL } \\
\hline 0 & 50,500 & 67,300 & 18 & 37,900 & 47,100 & 58,800 & 7 \\
\hline 2 & 51,300 & 67,700 & $171 / 2$ & 38,500 & 41,000 & 44,800 & $21 / 2$ \\
\hline 4 & 53,300 & 68,600 & 17 & 40,000 & 41,700 & 45,600 & 3 \\
\hline 8 & 61,500 & 70,200 & 9 & 46,100 & 53,500 & 57,900 & $21 / 2$ \\
\hline 16 & 63,400 & 69,200 & 6 & 47,600 & 56,500 & 61,000 & $31 / 2$ \\
\hline 24 & 63,500 & 69,700 & $61 / 2$ & 47,300 & 56,800 & 61,100 & 3 \\
\hline $\begin{array}{l}\text { Standard de- } \\
\text { viation. }\end{array}$ & 985 & 490 & 0.6 & -....... & 1,110 & 1,200 & 0.4 \\
\hline
\end{tabular}

1 Two of these specimens broke in corroding medium. 
TABLE 6.-Effect of aging period at $400^{\circ} \mathrm{F}$. on parallel tensile properties and resistance to corrosion of 0.064 -in.-gage commercial $24 \mathrm{~S}-\mathrm{T}$ aluminum alloy

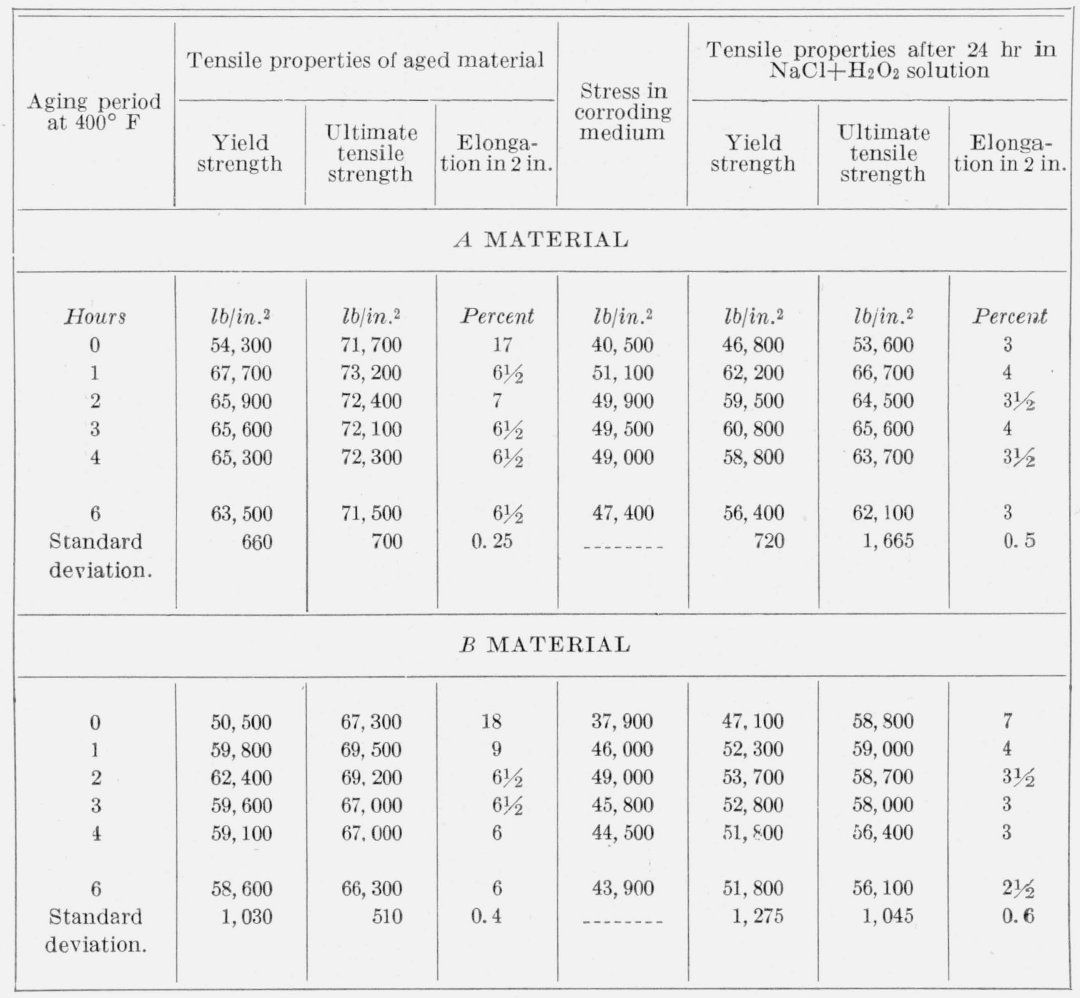




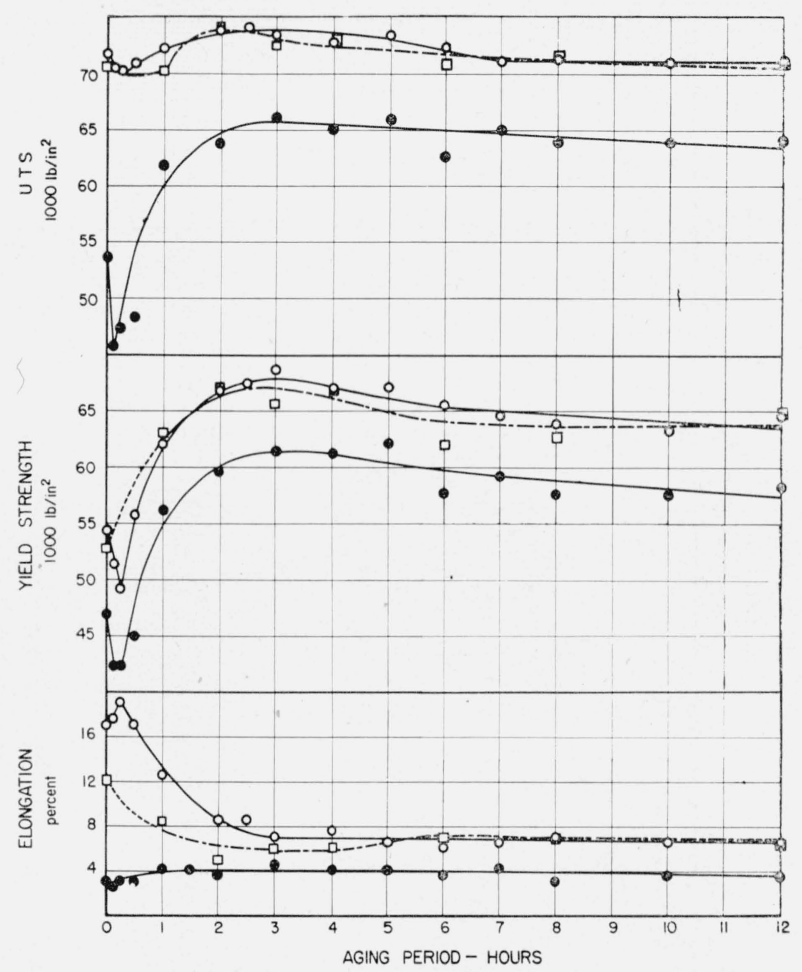

Figure 5.-Tensile properties of parallel specimens of $A$ material, aged at $385^{\circ} \mathrm{F}$, before and after exposure (anodized) to a marine atmosphere for 6 weeks and in the $\mathrm{NaCl}+\mathrm{H}_{2} \mathrm{O}_{2}$ solution without surface protection for $24 \mathrm{hr}$. $\bigcirc$, Uncorroded; $\square$, exposed in marine atmosphere, stressed to three-fourths of yield strength; corroded in $\mathrm{NaCl}+\mathrm{H}_{2} \mathrm{O}_{2}$ solution, stressed to threefourths of yield strength.

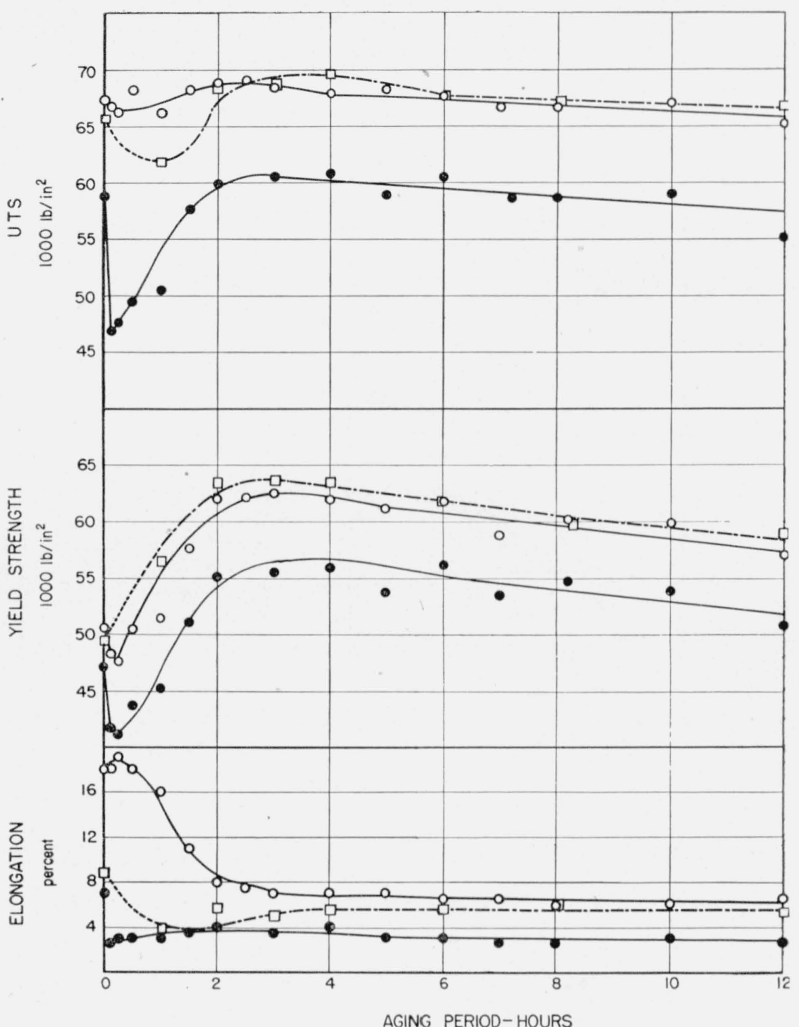

Figure 6.-Tensile properties of parallel specimens of $B$ material, aged at $385^{\circ} \mathrm{F}$, before and after exposure (anodized) to a marine atmosphere for 6 weeks and in the $\mathrm{NaCl}+\mathrm{H}_{2} \mathrm{O}_{2}$ solution without surface protection for $24 h r$.

$\bigcirc$, Uncorroded; $\square$, exposed in marine atmosphere, stressed to three-fourths of yield strength; corroded in $\mathrm{NaCl}+\mathrm{H}_{2} \mathrm{O}_{2}$ solution, stressed to threefourths of yield strength. 


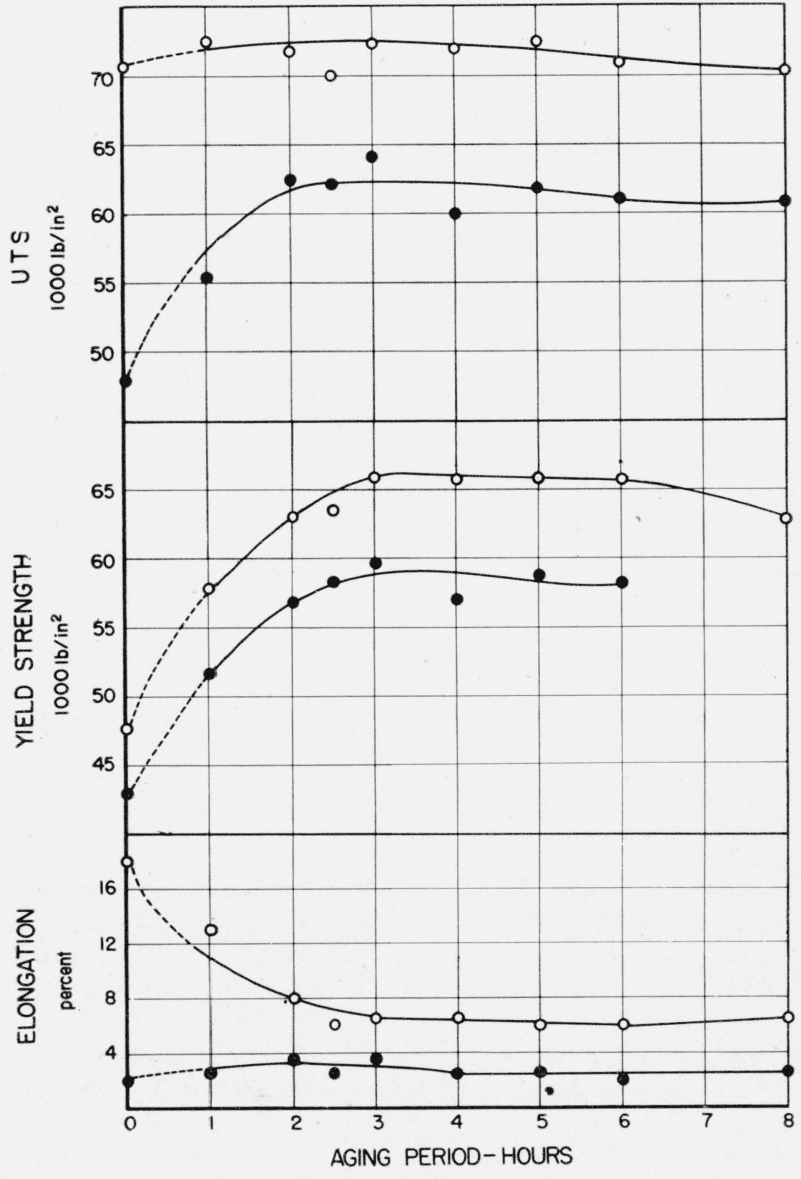

Figure 7.-Tensile properties of transverse specimens of A material, aged at $385^{\circ} \mathrm{F}$, before and after exposure for $24 \mathrm{hr}$ in the $\mathrm{NaCl}+\mathrm{H}_{2} \mathrm{O}_{2}$ solution.

$\mathrm{O}$, Uncorroded; $\boldsymbol{O}$, corroded in $\mathrm{NaCl}+\mathrm{H}_{2} \mathrm{O}_{2}$ solution, stressed to threefourths of yield strength.

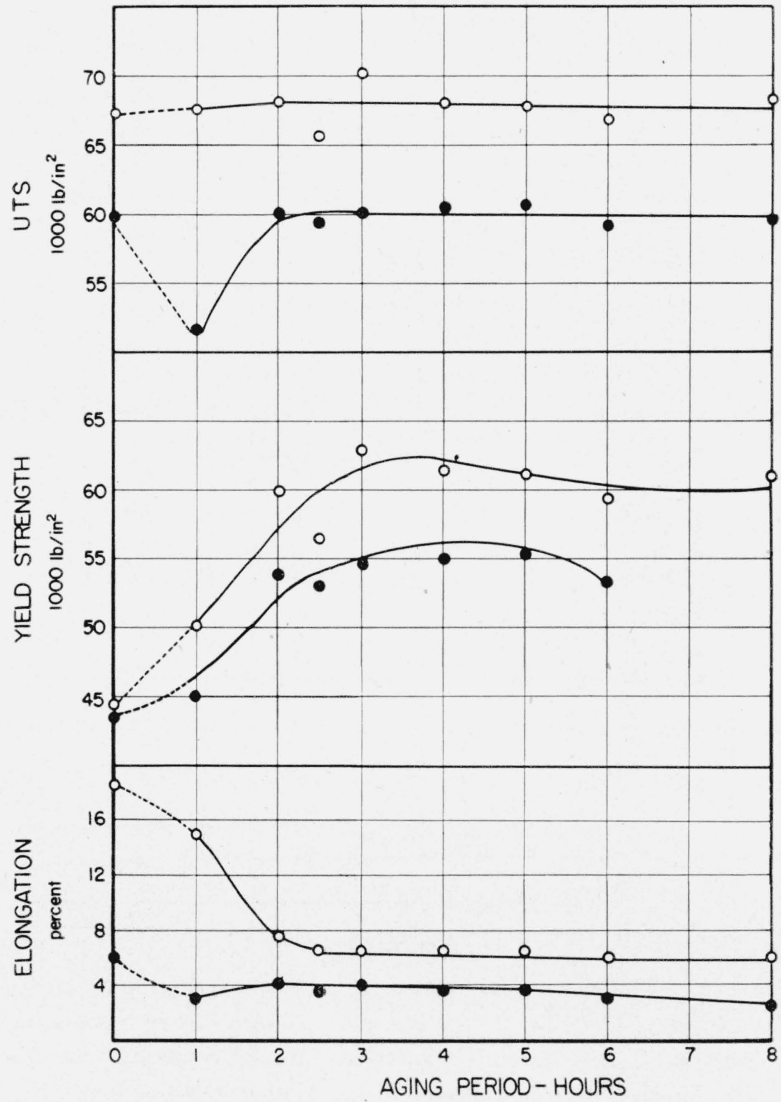

FIgURE 8.-Tensile properties of transverse specimens of $B$ material, aged at $385^{\circ} \mathrm{F}$, before and after exposure for $24 \mathrm{hr}$ in the $\mathrm{NaCl}+\mathrm{H}_{2} \mathrm{O}_{2}$ solution.

O, Uncorroded; - corroded in $\mathrm{NaCl}+\mathrm{H}_{2} \mathrm{O}_{2}$ solution, stressed to threefourths of yield strength. 


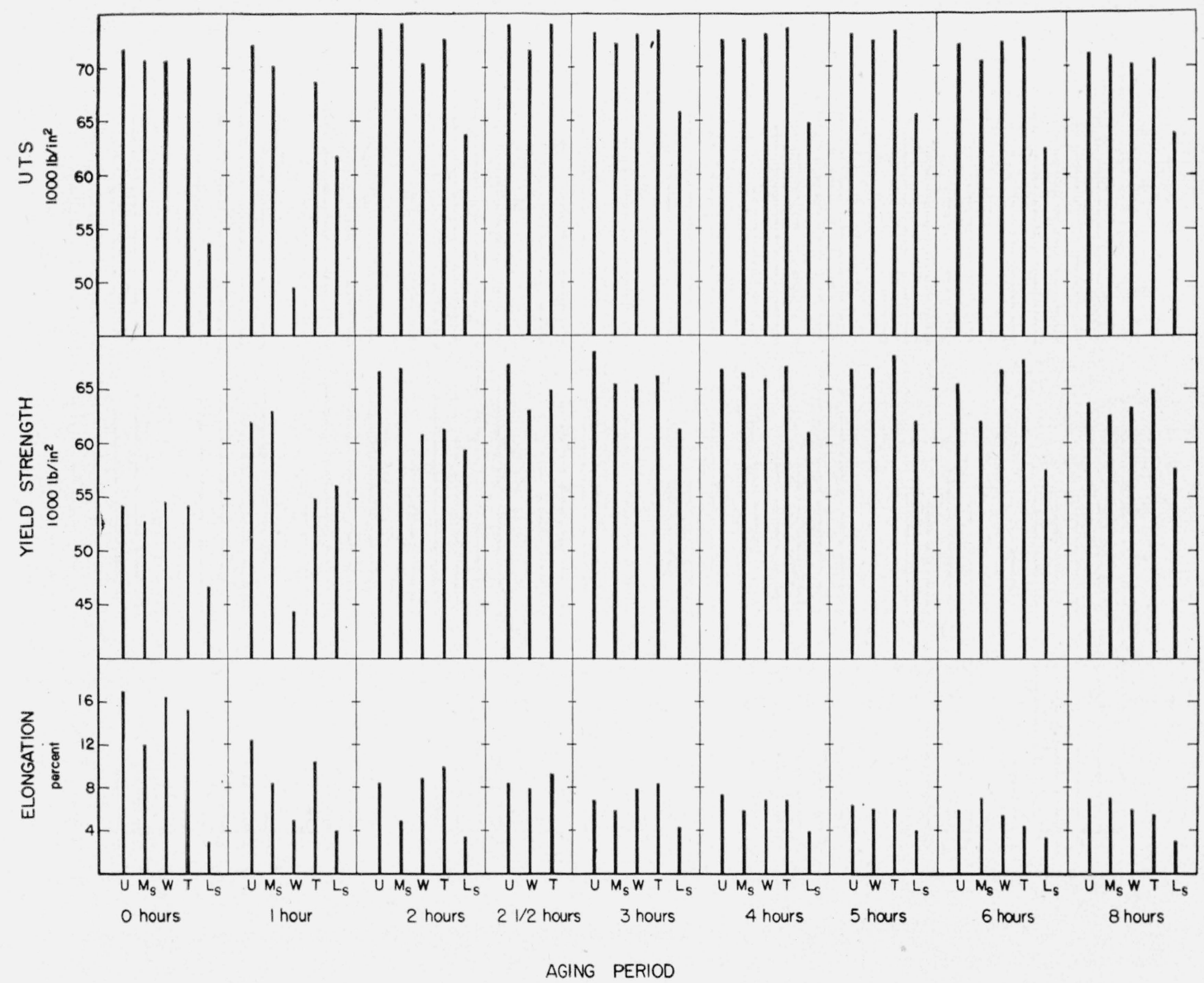

Frgure 9.-Tensile properties of parallel specimens of $\mathrm{A}$ material aged at $385^{\circ} \mathrm{F}$.

$U$, As aged; $M_{s}$, exposed, stressed to three fourths of yield strength, in marine atmosphere for 6 weeks; $W$, exposed, unstressed, in marine atmosphere for $1 \mathrm{yr} ; \mathrm{T}$, exposed, unstressed, to intermittent immersion in tidewater for 14 days; $L_{s}$, immersed, stressed to three fourths of yield strength, in the $\mathrm{NaCl}+\mathrm{H}_{2} \mathrm{O}_{2}$ solution in the laboratory for $24 \mathrm{hr}$. Specimens $M_{s}$ were anodized prior to exposure; all other specimens were exposed without surface protection. 


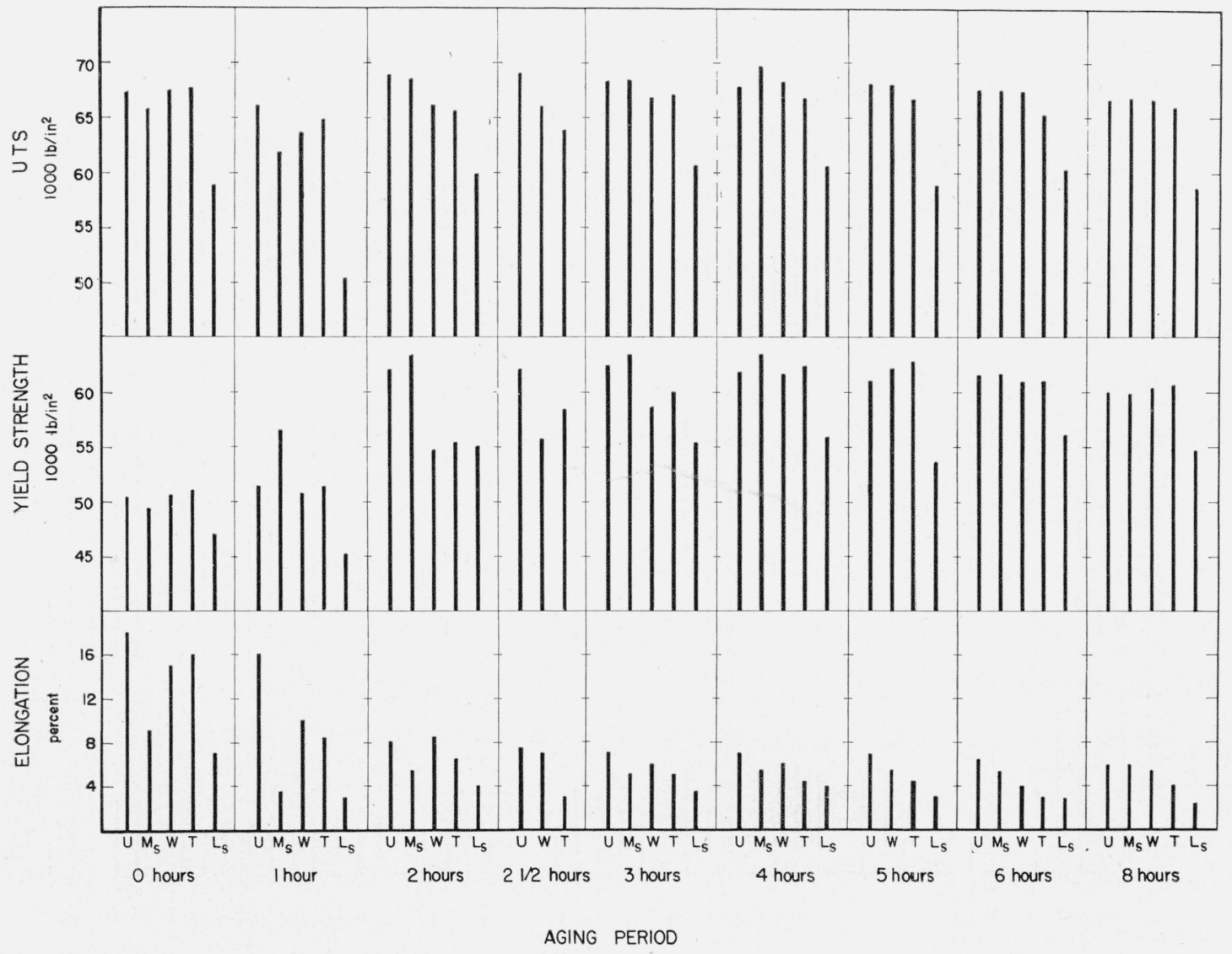

FIGURE 10.-Tensile properties of parallel specimens of $B$ material aged at $385^{\circ} \mathrm{F}$.

$U$, As aged; $M_{s}$, exposed, stressed to three fourths of yield strength, in marine atmosphere for 6 weeks; W, exposed, unstressed, in marine atmosphere for $1 \mathrm{yr} ; \mathrm{T}$, exposed, unstressed, to intermittent immersion in tidewater for 14 days; $L_{s}$, immersed, stressed to three fourths of yield strength, in the $\mathrm{NaCl}+\mathrm{H}_{2} \mathrm{O}_{2}$ solution in laboratory for $24 \mathrm{hr}$. Specimens $M_{s}$ were anodized prior to exposure, all other specimens were exposed without surface protection. 


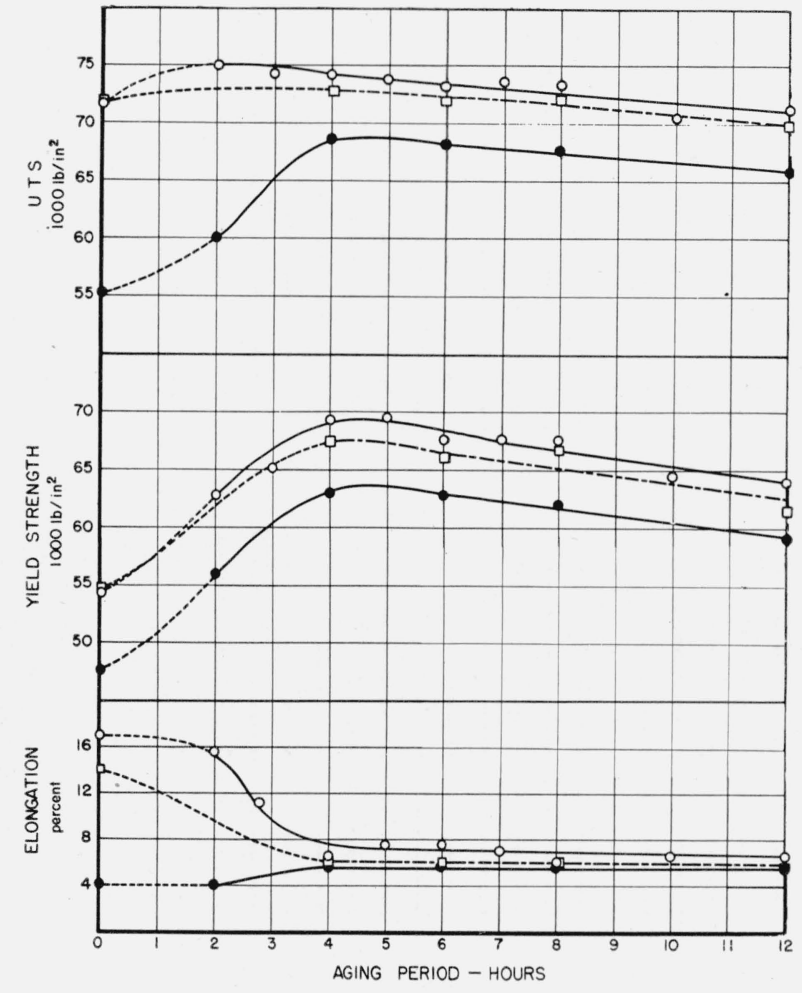

Figure 11.-Tensile properties of parallel A specimens, aged at $375^{\circ} \mathrm{F}$, before and after exposure in a marine atmosphere for 6 weeks and in the $\mathrm{NaCl}+\mathrm{H}_{2} \mathrm{O}_{2}$ solution for $24 \mathrm{hr}$.

$\bigcirc$, Uncorroded; $\square$, exposed in marine atmosphere, stressed to three-fourths of yield strength; - corroded in $\mathrm{NaCl}+\mathrm{H}_{2} \mathrm{O}_{2}$ solution, stressed to threefourths of yield strength.

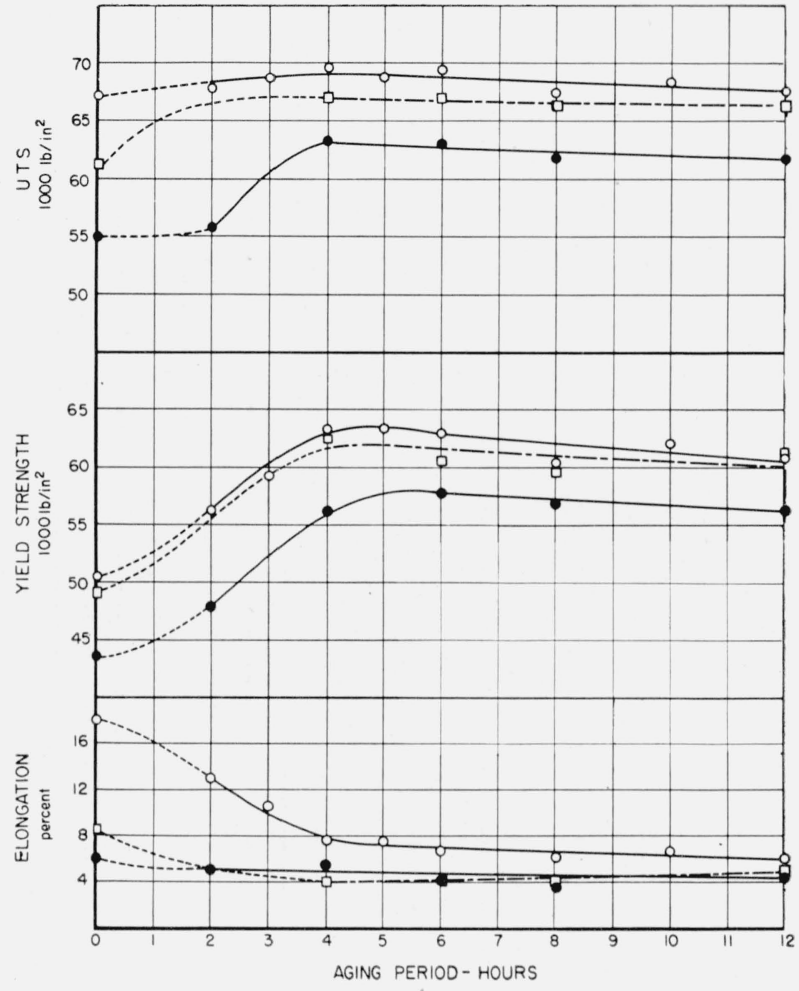

Figure 12.-Tensile properties of parallel B specimens, aged at $375^{\circ} \mathrm{F}$, before and after exposure in a marine atmosphere for 6 weeks and in the $\mathrm{NaCl}+\mathrm{H}_{2} \mathrm{O}_{2}$ solution for $24 \mathrm{hr}$.

$\bigcirc$, Uncorroded; $\square$, exposed in marine atmosphere, stressed to three-fourths of yield strength; - corroded in $\mathrm{NaCl}+\mathrm{H}_{2} \mathrm{O}_{2}$ solution, stressed to threefourths of yield strength. 


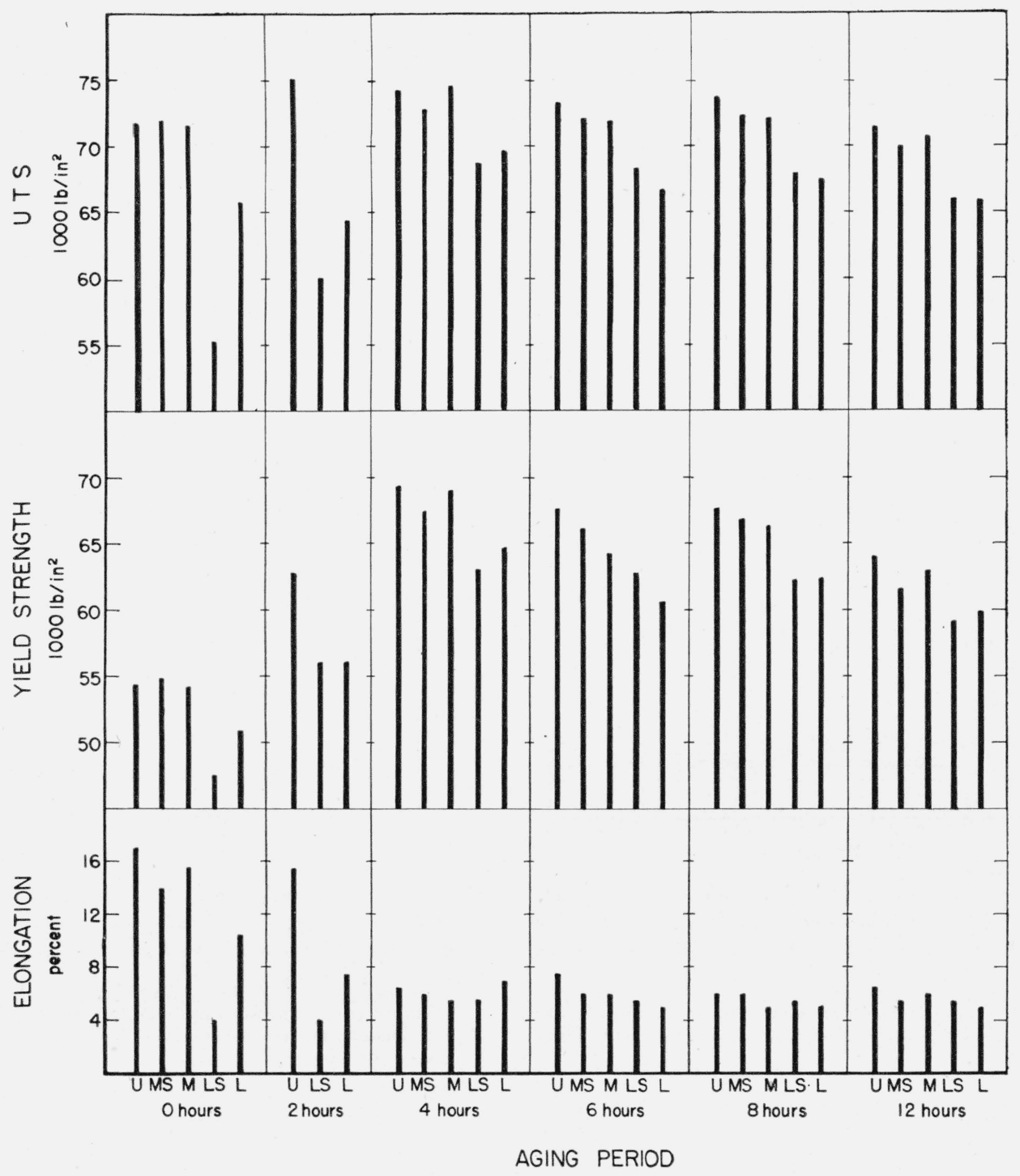

Figure 13.-Effect of stress and corrosion on tensile properties of parallel A specimens aged at $375^{\circ} \mathrm{F}$.

$U$, as aged; $M S$, corrodent, marine atmosphere, stressed to three-fourths of yield strength; $M$, corrodent, marine atmosphere, stress $=0 ; L S$, corrodent, $\mathrm{NaCl}+\mathrm{H}_{2} \mathrm{O}_{2}$, stressed to three-fourths of yield strength; $L$, corrodent, $\mathrm{NaCl}+\mathrm{H}_{2} \mathrm{O}_{2}$, stress $=0$. 


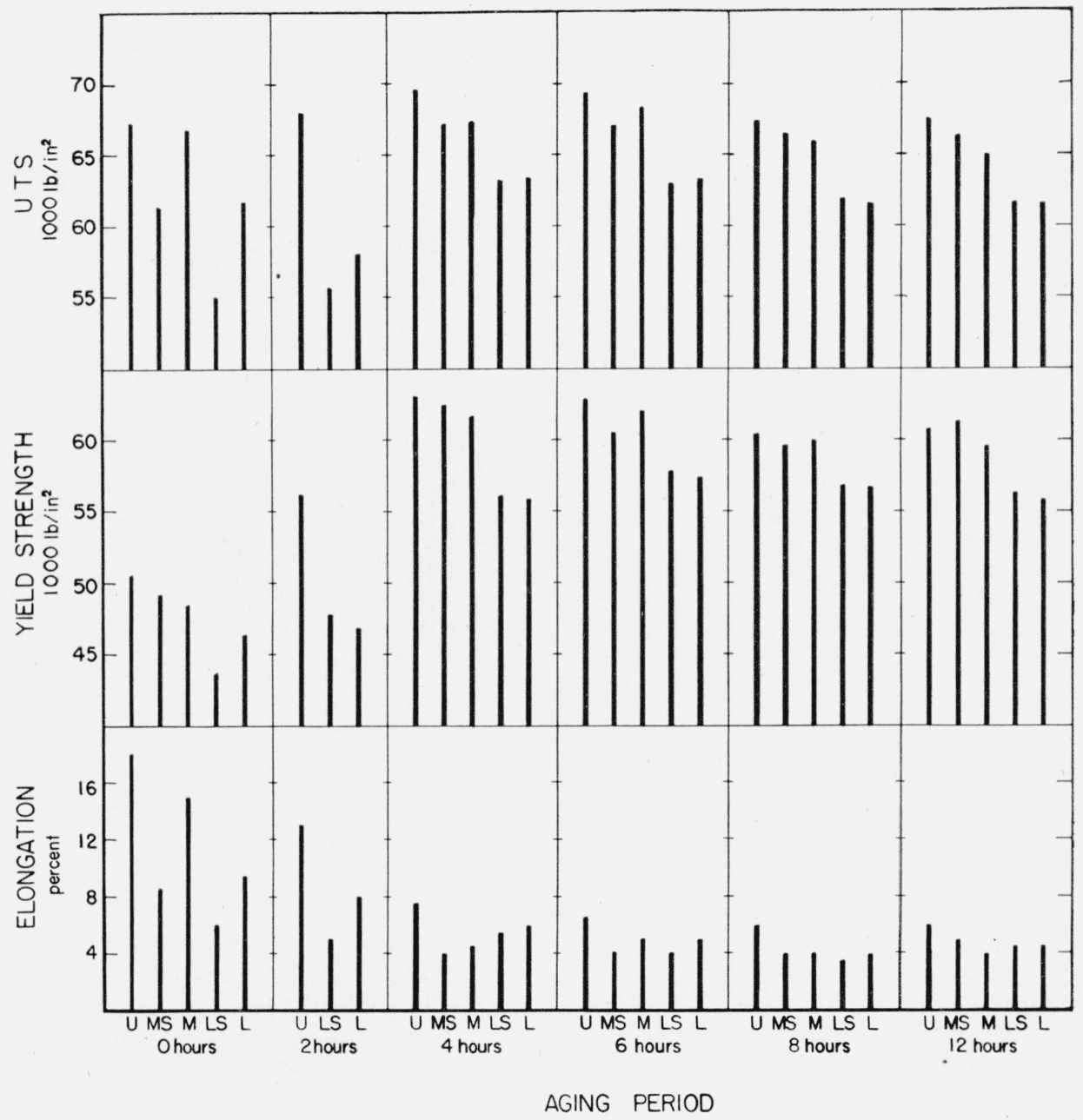

FiguRE 14.-Effect of stress and corrosion on tensile properties of parallel B specimens aged at $375^{\circ} \mathrm{F}$.

$U$, as aged; MS, corrodent, marine atmosphere, stressed to three-fourths of yield strength; $M$, corrodent, marine atmosphere, stress $=0$; $L S$, corrodent, $\mathrm{NaCl}+\mathrm{H}_{2} \mathrm{O}_{2}$, stressed to three-fourths of yield strength; $L$, corrodent, $\mathrm{NaCl}+\mathrm{H}_{2} \mathrm{O}_{2}$, stress $=0$. 


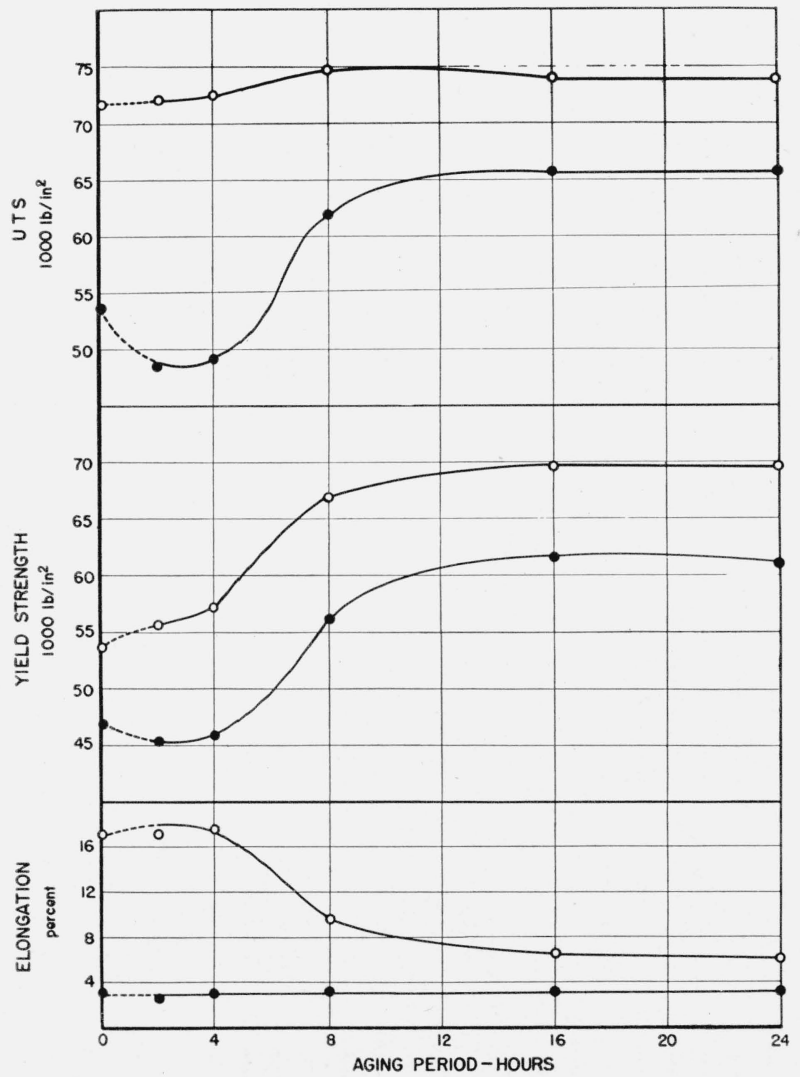

FIGURE 15.-Tensile properties of parallel A specimens, aged at $350^{\circ} \mathrm{F}$, before and after exposure for $24 \mathrm{hr}$ in the $\mathrm{NaCl}+\mathrm{H}_{2} \mathrm{O}_{2}$ solution.

$\mathrm{O}$, Uncorroded; - corroded in $\mathrm{NaCl}+\mathrm{H}_{2} \mathrm{O}_{2}$ solution, stressed to threefourths of yield strength.

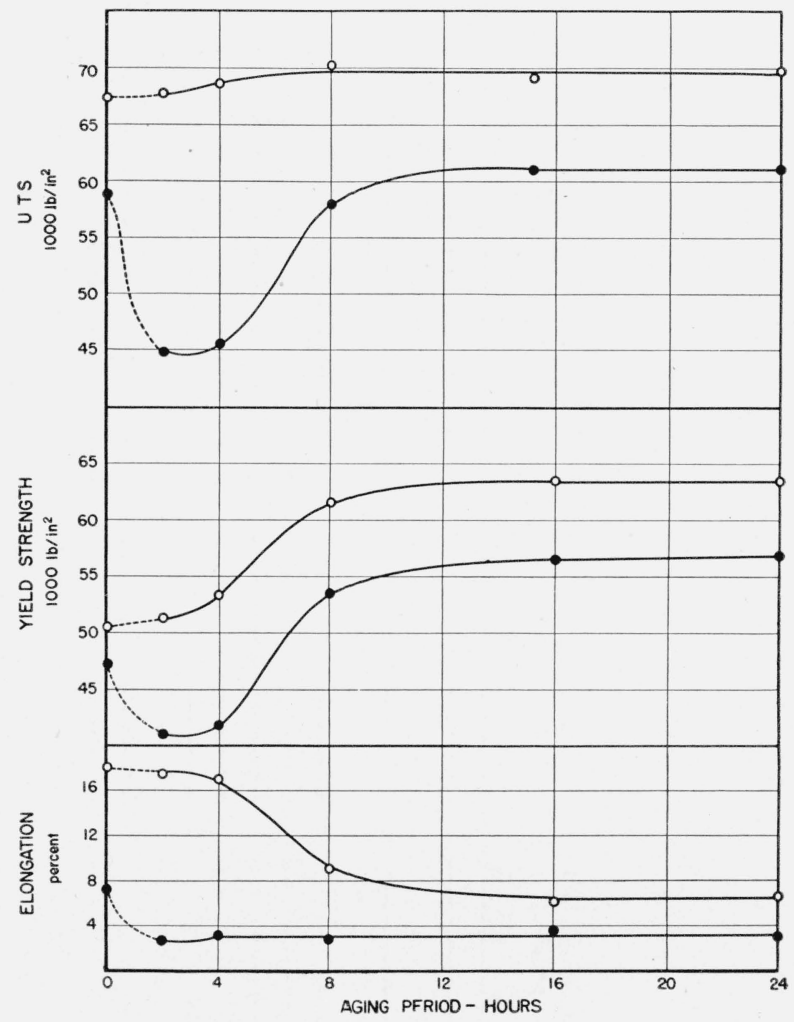

Figure 16.--Tensile properties of parallel $B$ specimens, aged at $350^{\circ} \mathrm{F}$, before and after exposure for $24 \mathrm{hr}$ in the $\mathrm{NaCl}+\mathrm{H}_{2} \mathrm{O}_{2}$ solution.

$\mathrm{O}$, Uncorroded; , corroded in $\mathrm{NaCl}+\mathrm{H}_{2} \mathrm{O}_{2}$ solution, stressed to threefourths of yield strength. 


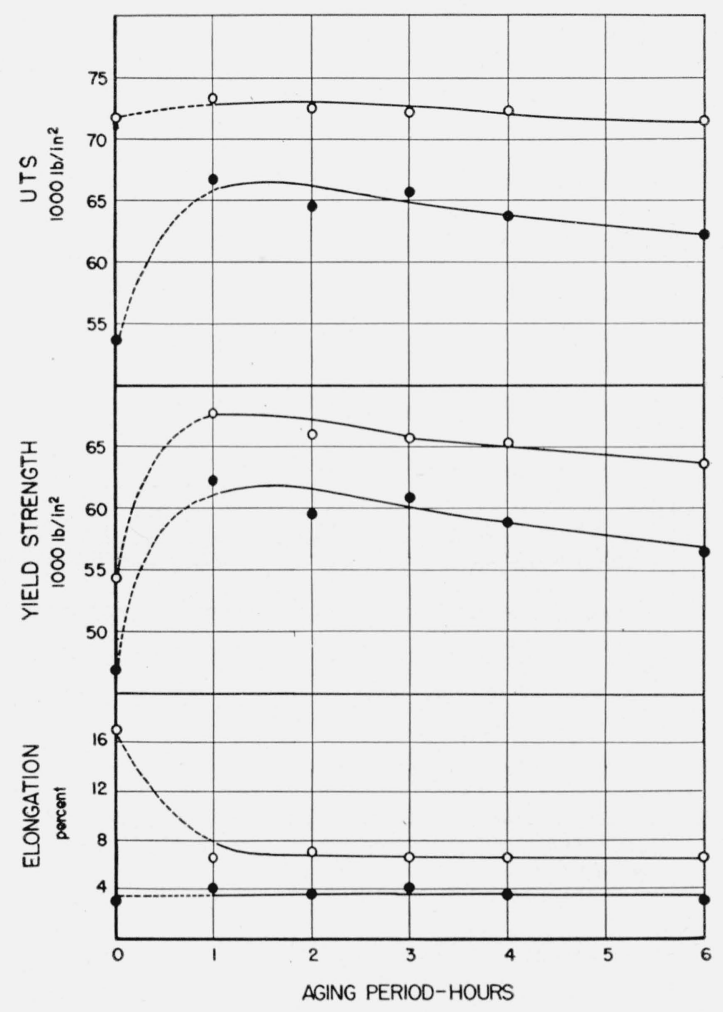

Figure 17.-T'ensile properties of parallel A specimens, aged at $400^{\circ} \mathrm{F}$, before and after exposure for $24 \mathrm{hr}$ in the $\mathrm{NaCl}+\mathrm{H}_{2} \mathrm{O}_{2}$ solution.

$\bigcirc$, Uncorroded; $\boldsymbol{O}$, corroded in $\mathrm{NaCl}+\mathrm{H}_{2} \mathrm{O}_{2}$ solution, stressed to threefourths of yield strength.

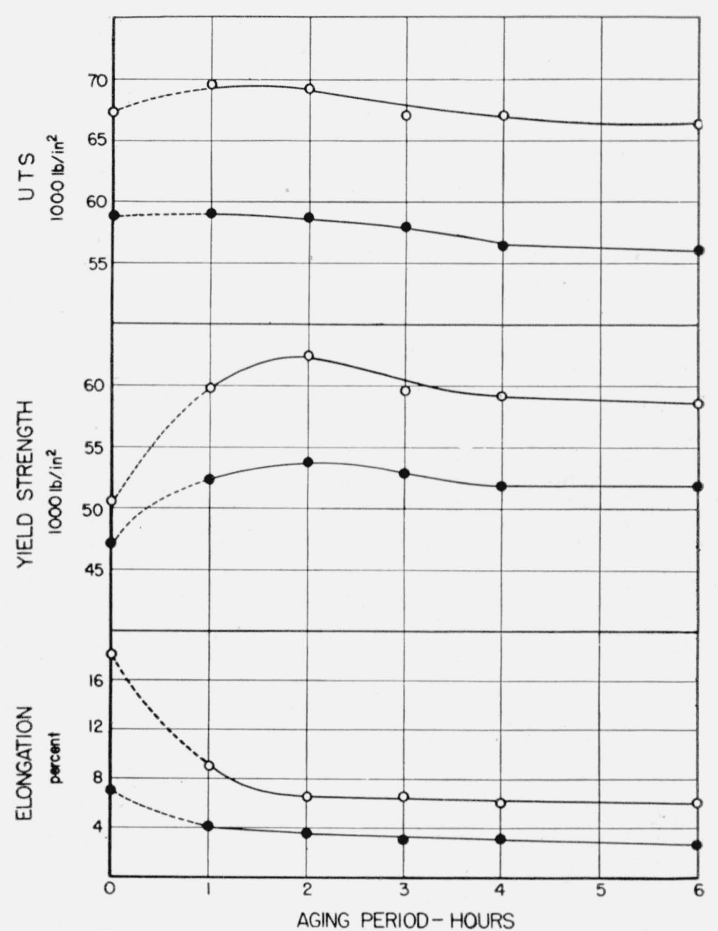

FIgURE 18.-Tensile properties of parallel $B$ specimens, aged at $400^{\circ} \mathrm{F}$, before and after exposure for $24 \mathrm{hr}$ in the $\mathrm{NaCl}+\mathrm{H}_{2} \mathrm{O}_{2}$ solution.

, Uncorroded; - corroded in $\mathrm{NaCl}+\mathrm{H}_{2} \mathrm{O}_{2}$ solution, stressed to threefourths of yield strength. 


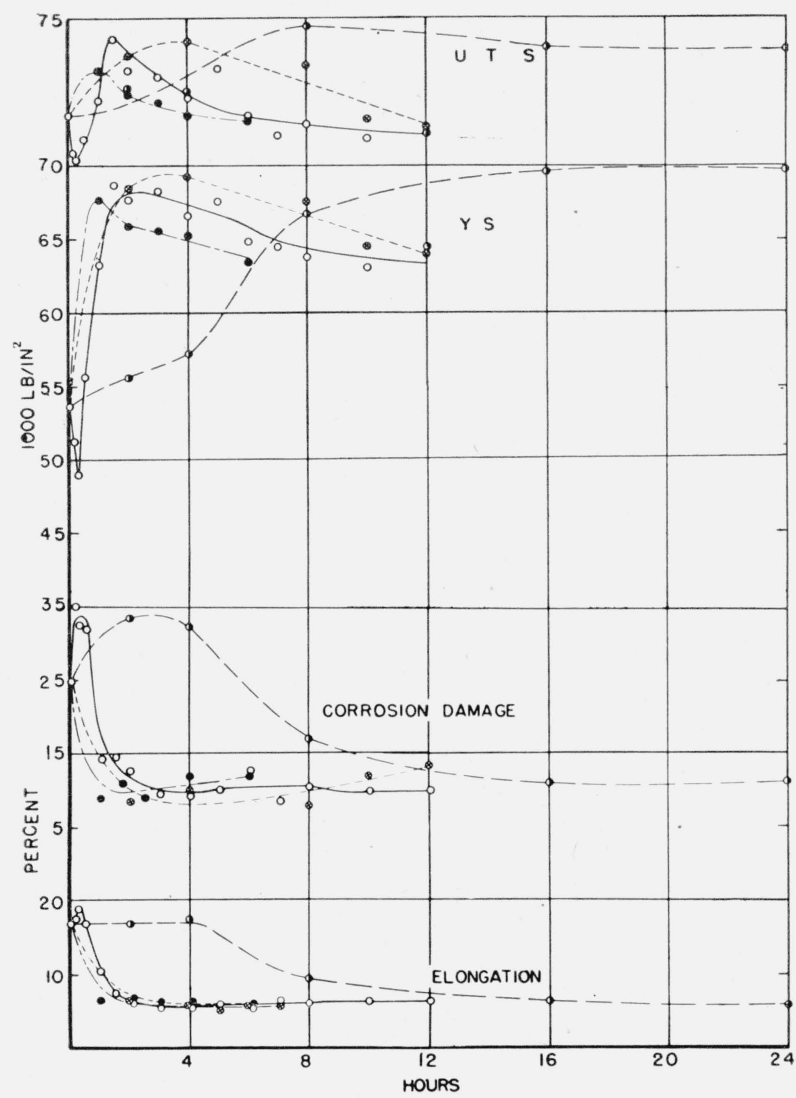

Figure 19,--Relationship between aging time and tensile properties of parallel A specimens.

Corrosion damage $=$ percentage loss in ultimate tensile strength of specimens exposed for $24 \mathrm{hr}$ in the $\mathrm{NaCl}+\mathrm{H}_{2} \mathrm{O}_{2}$ solution under stress equal to threefourths of yield strength. Aging temperature: $350^{\circ} \mathrm{F} ; \oplus, 375^{\circ} \mathrm{F} ; 0$; $385^{\circ} \mathrm{F} ; 0,400^{\circ} \mathrm{F}$.

\section{Effect of Aging on Tensile Properties}

The aging period necessary to obtain the maximum values for tensile and yield strengths varied from approximately $20 \mathrm{hr}$ at $350^{\circ} \mathrm{F}$ to $1 \frac{1}{2} \mathrm{hr}$ at $400^{\circ} \mathrm{F}$.

\section{(a) Materials Aged at $385^{\circ} \mathrm{F}$}

The results for parallel specimens are shown in figures 5 and 6 and in tables 1 and 2. Aging of the materials for periods of 8 and 15 minutes reduced the ultimate tensile and yield strengths and increased the percentage elongations. For longer aging periods the yield and ultimate tensile strengths increased and reached maximum values, for $2 \frac{1}{2}$ to $3 \mathrm{hr}$ aging, as follows: Ultimate tensile strength $74,100 \mathrm{lb} /$ in. $^{2}$ and $69,000 \mathrm{lb} / \mathrm{in}^{2}$, for the $A$ and $B$ lots of material respectively; the maximum yield strengths were $68,600 \mathrm{lb} / \mathrm{in}^{2}$ and $62,500 \mathrm{lb} / \mathrm{in}^{2}$, respectively. The values for

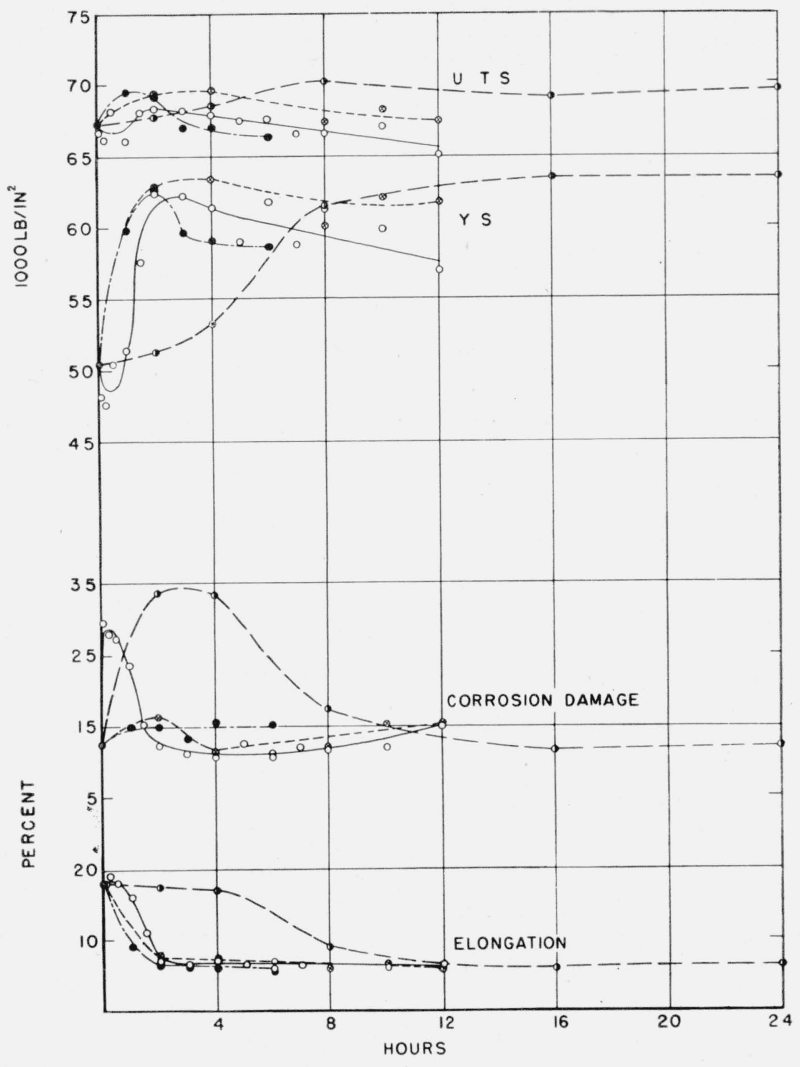

Figure 20.-Relationship between aging time and tensile properties of parallel $B$ specimens.

Corrosion damage $=$ percentage loss in ultimate tensile strength of specimens exposed for $24 \mathrm{hr}$ in the $\mathrm{NaCl}+\mathrm{H}_{2} \mathrm{O}_{2}$ solution under stress equal to three-fourths of yield strength. Aging temperature: $350^{\circ} \mathrm{F} ; \oplus, 375^{\circ} \mathrm{F}$; $\bigcirc, 385^{\circ} \mathrm{F} ; 400^{\circ} \mathrm{F}$.

these properties decreased slightly as the aging periods were increased to $12 \mathrm{hr}$. The percentage elongation decreased as the aging period was increased beyond 15 minutes and became constant, at $6 \frac{1 / 2}{2}$ to 7 percent, after aging periods of 3 to 6 hours.

Computation of the standard deviations for aging periods that may be commercially important ( $2 \frac{1}{2}$ to $6 \mathrm{hr}$, inclusive) gave values somewhat smaller than those obtained from the averages of the ranges of all the aging periods. The standard deviations over this range for the yield strengths were $810 \mathrm{lb} /$ in. $^{2}$ and $565 \mathrm{lb} / \mathrm{in}^{2}$; for the ultimate tensile strengths, $390 \mathrm{lb} / \mathrm{in}^{2}{ }^{2}$ and $590 \mathrm{lb} / \mathrm{in}^{2}{ }^{2}$; and for the percentage elongations, 0.3 and 0.25 percent for the $A$ and $B$ materials, respectively.

The results obtained on transverse specimens are summarized in figures 7 and 8 and in tables 1 and 2. The maximum yield strengths were 
obtained by aging for $3 \mathrm{hr}$. The ultimate tensile strengths were increased 2 to $4 \frac{1}{2}$ percent by aging at this temperature. The original yield strengths of the transverse specimens were lower than those of the parallel specimens, and the yield and tensile strengths of the transverse $A$ specimens after aging periods of $3 \mathrm{hr}$ or longer were generally 1,000 to $3,000 \mathrm{lb} / \mathrm{in}^{2}{ }^{2}$ lower than those of the parallel specimens aged for the same periods. However, the values of the tensile properties of the $B$ material were approximately the same, after aging periods of $3 \mathrm{hr}$ or more, whether the specimens had been taken transversely or parallel to the direction of rolling.

(b) Materials aged at $350^{\circ}, 375^{\circ}$, and $400^{\circ} \mathrm{F}$

Data for aging periods at $375^{\circ}, 350^{\circ}$, and $400^{\circ}$ $\mathrm{F}$ are shown graphically in figures 11 to 18 , inclusive, and are given in tables 4,5 , and 6 . Rather complete data were obtained for aging periods of 2 to $12 \mathrm{hr}$ at $375^{\circ} \mathrm{F}$, but at $350^{\circ}$ and $400^{\circ} \mathrm{F}$ the specimens were aged at only enough periods to give the general shape of the curves of tensile properties plotted against aging periods.

Maximum values for the yield strength were obtained by aging the materials for from 4 to 6 $\mathrm{hr}$ at $375^{\circ} \mathrm{F}$ and from 16 to $24 \mathrm{hr}$ at $350^{\circ} \mathrm{F}$. No specimens were aged for a period of less than 1 hr at $400^{\circ} \mathrm{F}$; data on the $A$ specimens indicate that the maximum yield strength for this material might possibly have been obtained by aging this material for less than $1 \mathrm{hr}$.

Irrespective of the aging temperatures there was a maximum increase of approximately 3 percent in ultimate tensile strengths of parallel specimens over that of the unaged material and 25 percent in yield strengths. The increases in yield and ultimate tensile strengths were accompanied by decreases of about 60 percent in the values for percentage elongation.

Standard deviations were approximately the same whether the values of all the specimens aged at $375^{\circ} \mathrm{F}$ or only those aged for periods of 4 to $12 \mathrm{hr}$ were considered. Standard deviations for all the data were computed for specimens aged at $350^{\circ}$ and $400^{\circ} \mathrm{F}$.

\section{Susceptibility of Materials to Corrosion}

It was found that, in general, specimens aged for relatively short periods of time were the most severely damaged in the corroding media; as the aging period was increased to produce the maximum yield strength, the materials became less susceptible to corrosion and stress-corrosion cracking. Further aging, up to four times the period necessary to produce maximum properties for specimens aged at $385^{\circ} \mathrm{F}$, did not appreciably change the resistance of the specimens to corrosion.

(a) Materials Aged at $385^{\circ} \mathrm{F}$

In the laboratory exposures of stressed specimens in $\mathrm{NaCl}+\mathrm{H}_{2} \mathrm{O}_{2}$ solution, the changes in ultimate tensile strength and percentage elongation of materials aged for 8,15 , and 30 minutes, and subsequently immersed for $24 \mathrm{hr}$ in the $\mathrm{NaCl}+\mathrm{H}_{2} \mathrm{O}_{2}$ solution, were much greater than those of specimens aged $1 \mathrm{hr}$ or longer. The resistance of the material to the combined action of stress and corrosion continued to increase as the aging period was increased from 1 to $3 \mathrm{hr}$. Thereafter, there was little if any change in corrosion resistance with increased aging periods. (See figs. 5 to 10 and tables 1 to 3 .)

Specimens of commercially heat-treated material and specimens that had been aged for 1, 2, $3,4,6,8$, and $12 \mathrm{hr}$ at $385^{\circ} \mathrm{F}$ were stressed to three-fourths of the yield strength and exposed in the marine atmosphere. Unaged specimens and specimens aged for $1 \mathrm{hr}$ were more severely damaged than specimens aged for longer periods prior to exposure. Material aged $3 \mathrm{hr}$ or longer was, in general, less severely damaged after 6 weeks of exposure under stress in the marine atmosphere than the unaged material exposed under the same conditions.

The results obtained with unstressed panels exposed to the weather in a marine atmosphere and to alternate immersion in tidewater were in agreement with those given above. The greatest losses in tensile strength and ductility (see figs. 9 and 10 and table 3 ) occurred in materials aged less than $3 \mathrm{hr}$. In the weather exposure, specimens of both materials aged $3 \mathrm{hr}$ or longer were generally less severely damaged than the unaged materials. This was also true of the $A$ material in tidewater, but the unaged $B$ material proved, in general, to be slightly more resistant to corrosion in tidewater than any of the aged $B$ material.

Examination of the microstructures of specimens aged for various periods of time and subsequently exposed in the various corrosive media showed that material aged 8 minutes or longer 
was susceptible to intercrystalline corrosion in some degree. Specimens aged 8 minutes to $1 \mathrm{hr}$ and subsequently exposed to the $\mathrm{NaCl}+\mathrm{H}_{2} \mathrm{O}_{2}$ solution contained severe intercrystalline corrosion and the beginnings of stress corrosion cracks, as shown in figure $21, \mathrm{~A}$. A material aged for a period of $1 \mathrm{hr}$ and exposed unstressed in a marine atmosphere for 1 year contained more severe intercrystalline corrosion than was found in any other material examined (fig. 21, B). With longer aging periods, the areas of intercrystalline attack became less general and more spotty (fig. 21, C). For aging periods of 8 to $12 \mathrm{hr}$, the intercrystalline corrosion was associated with pitting (fig. 21, D).

\section{(b) Effect of Aging at $375^{\circ} \mathrm{F}$}

Unaged specimens and specimens that had been aged for $2 \mathrm{hr}$ were more severely damaged when exposed under stress in the $\mathrm{NaCl}+\mathrm{H}_{2} \mathrm{O}_{2}$ solution than similar specimens exposed under identical conditions except that they were not stressed. The effect of stress in increasing corrosion damage was less pronounced in specimens aged $4 \mathrm{hr}$ than in specimens aged for the shorter periods and there was generally little difference in corrosion damage to specimens aged $6 \mathrm{hr}$ or longer whether the specimens had been exposed stressed or unstressed in the corroding medium.

The losses in tensile strength and ductility of materials aged $6 \mathrm{hr}$ or longer and subsequently exposed for 6 weeks in a marine atmosphere were generally no greater than those for the commercially heat treated but unaged material.

\section{(c) Effect of Aging at $350^{\circ} \mathrm{F}$}

Metallographic examinations of specimens after their removal from the $\mathrm{NaCl}+\mathrm{H}_{2} \mathrm{O}_{2}$ solution indicated that all the aged material contained intercrystalline corrosion in some degree. Specimens aged for periods up to $12 \mathrm{hr}$ contained more severe intercrystalline corrosion than specimens aged for longer periods of time.

\section{(d) Effect of Aging at $400^{\circ} \mathrm{F}$}

Material aged $2 \mathrm{hr}$ or longer contained only traces of intercrystalline corrosion on metalloographic examination of specimens after their their removal from the $\mathrm{NaCl}+\mathrm{H}_{2} \mathrm{O}_{2}$ corroding solution.
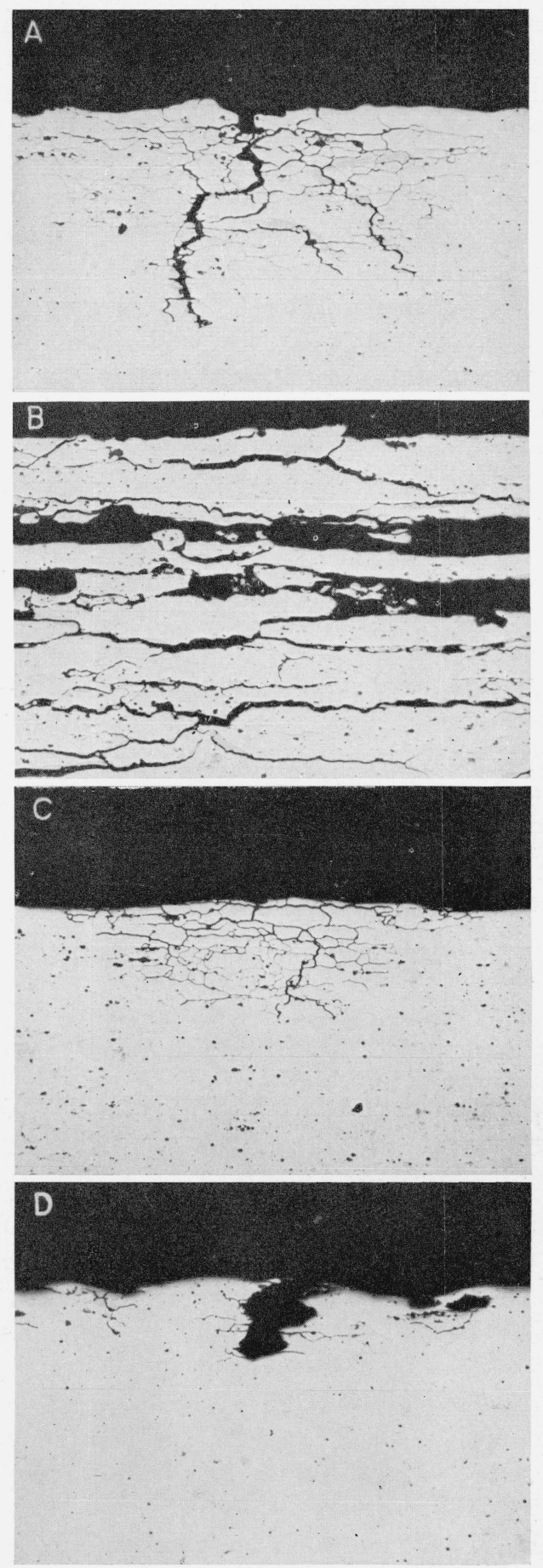

FIGURE 21.-Types of corrosion.

A, Severe, intercrystalline, and beginning of stress corrosion crack typical of corrosion produced in material aged about 30 minutes at $385^{\circ} \mathrm{F}$. Unetched. $\times 100 ; B$, material aged $1 \mathrm{hr}$ and exposed (unstressed) for $1 \mathrm{yr}$ in marine atmosphere. Unetched. $\times 100 ; C$, less severe intercrystalline than that shown in $A$ and typical of corrosion found in material aged for longer periods ( 3 to $4 \mathrm{hr}$ at $385^{\circ} \mathrm{F}$ ) prior to exposure. Unetched. $\times 100 ; D$, intercrystalline extending out from pits. Typical of corrosion found in material aged for 8 to $10 \mathrm{hr}$ at $385^{\circ} \mathrm{F}$ prior to exposure. Unetched. $\times 100$. 


\section{Discussion of results}

The changes in tensile properties with the aging periods, for the four temperatures used are shown in figures 19 and 20. As was indicated above, approximately the same maximum tensile properties were obtained for the various aging temperatures used between $350^{\circ}$ and $400^{\circ} \mathrm{F}$. However, the aging period necessary to produce the maximum yield strength decreased with increased aging temperatures. In many metallurgical phenomena, particularly where diffusion may be involved, it is found that a linear relation holds between the logarithm of the time and the reciprocal of the absolute temperature. Accordingly the reciprocals of the aging temperatures in degrees Kelvin were plotted as ordinates, and the aging times necessary to obtain maximum yield strengths were plotted on a logarithmic scale, as abscissas in figure 22. The plotted points lie approximately on a straight line represented by an equation of the form

$$
t=A e^{-} \frac{B}{K}
$$

where $t$ is the aging period in hours, $K$ the absolute temperature in degrees $\mathrm{K}$, and $A$ and $B$ constants whose values are

$$
A=3.85 \times 10^{-19} \mathrm{hr}, \quad B=-20.4 \times 10^{3} \text { degrees } \mathrm{K} \text {. }
$$

The aging temperatures in degrees Fahrenheit were also plotted against the aging periods for maximum yield strength as shown in figure 22 . A curve was obtained similar to that shown by Mozley [1] but with a somewhat different slope. Better correlation was evident, between the curve (computed by the method of least squares) and the plotted points, when aging periods were plotted against the reciprocals of the absolute temperatures than against the Fahrenheit temperatures. The Bravais-Pearson coefficients of correlation were 0.999 and 0.866 , respectively, for the two curves.

The $24 \mathrm{~S}-\mathrm{T}$ alloy transferred without delay from the solution heat-treating furnace to the quenching bath and cooled at a sufficiently high rate [3] to room temperature is immune to the very damaging intercrystalline type of corrosion and is susceptible only to the more innocuous pitting type of corrosion. It is generally considered that the susceptibility of the material to

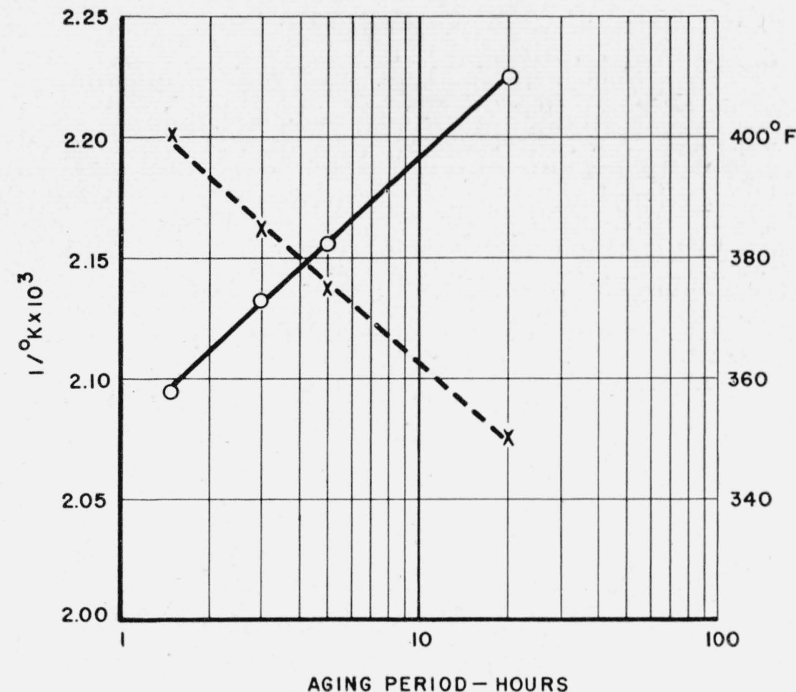

Figure 22.-Relationship between aging temperature and aging period necessary to produce maximum yield strengths.

Rising line represents reciprocals of absolute temperatures, and falling line represents temperature in degrees $\mathrm{F} . \quad \mathrm{O}, 1 /{ }^{\circ} \mathrm{K}$-time; $\times,{ }^{\circ} \mathrm{F}$-time.

corrosion damage is increased by elevated temperature aging as well as by failure to satisfy the above conditions for rapid cooling. The results of the corrosion and stress-corrosion tests in the laboratory, in a marine atmosphere and in tidewater were significant in that they show: (a) that material that had been aged for a period sufficient to produce maximum mechanical properties was, in general, no more susceptible to corrosion damage than the commercially heat-treated material exposed without elevated-temperature aging, (b) that the application of stresses, equal to threefourths of the yield strength, did not appreciably increase corrosion damage, over that to unstressed material, for specimens aged $6 \mathrm{hr}$ or longer at $375^{\circ} \mathrm{F}$. Stressed specimens of materials exposed after an aging period of $2 \mathrm{hr}$ at $375^{\circ} \mathrm{F}$, and specimens exposed with no elevated-temperature aging were generally much more severely damaged than the comparable specimens exposed unstressed in the corroding media, (c) there were no data to indicate that material aged at one temperature, in the range $350^{\circ}$ to $400^{\circ} \mathrm{F}$, sufficiently long to obtain the maximum physical properties was more resistant to corrosion than material aged at another temperature to the same condition. 


\section{Summary}

1. Commercially heat-treated $24 \mathrm{~S}-\mathrm{T}$ aluminum alloy sheet (0.064 in. thick) was artificially aged for various periods at $350^{\circ}, 375^{\circ}, 385^{\circ}$, and $400^{\circ} \mathrm{F}$. The tensile properties resulting from artificial aging and the resistance of the aged material to stress-corrosion cracking in $\mathrm{NaCl}+\mathrm{H}_{2} \mathrm{O}_{2}$ solution and (for some of the materials) in a marine atmosphere, were determined.

2. The maximum yield and ultimate tensile strengths obtained in this alloy were independent of the aging temperature between $350^{\circ}$ and $400^{\circ} \mathrm{F}$. The optimum results were obtained by aging the material approximately $20 \mathrm{hr}$ at $350^{\circ} \mathrm{F}, 5 \mathrm{hr}$ at $375^{\circ} \mathrm{F}, 3 \mathrm{hr}$ at $385^{\circ} \mathrm{F}$, and $1 \frac{1}{2} \mathrm{hr}$ at $400^{\circ} \mathrm{F}$.

3 . The effect of the aging treatment at $385^{\circ} \mathrm{F}$ was more pronounced on the tensile properties in a transverse direction than on those in a direction parallel to the direction of rolling. The yield strength of the transverse specimens of the commercially heat-treated material was increased approximately 40 percent above an initial value of about $45,000 \mathrm{lb} / \mathrm{in}^{2}$, and the ultimate tensile strength approximately 3 percent above an initial value of about $70,000 \mathrm{lb} / \mathrm{in} .^{2}$ The percentage elongation was reduced to about one-third of the initial value of 18 . Effects of the same magnitude were observed for the parallel specimens, except that the yield strength was increased only about 25 percent above an initial value of about 50,000 $\mathrm{lb} / \mathrm{in}^{2}$.

4. Corrosion damage was measured by losses in elongation and ultimate tensile strength. Elevatedtemperature aging of materials for short periods compared to those necessary to obtain the maximum mechanical properties, increased the susceptibility of the material to corrosion. However, for aging periods sufficiently long ${ }^{3}$ to produce maximum physical properties, the resistance to corrosion of the artificially aged material was generally at least equal to that of the commercially heattreated material exposed without elevated temperature aging. The ultimate tensile strengths of specimens exposed under stress in the sodium chloride-hydrogen peroxide solution were reduced as much as 30 to 35 percent for material aged for short periods of time. The reduction was only 10

\footnotetext{
${ }^{3}$ In some instances, also for aging periods up to three or four times the minimum necessary to produce maximum physical properties.
}

to 15 percent for specimens aged for periods sufficiently long to produce maximum tensile properties. The same amount of exposure of unaged material reduced its ultimate tensile strength by 11 to 32 percent.

Losses in tensile strength and ductility of anodized material aged $3 \mathrm{hr}$ or longer at $385^{\circ} \mathrm{F}$ and exposed for 6 weeks under stress in the marine atmosphere were small; the maximum loss in ultimate tensile strength was $1,500 \mathrm{lb} / \mathrm{in} .^{2}$, a loss which is probably not significant in this case; the maximum loss in ductility was represented by specimens in which the percent elongation was reduced from 7 to 5 .

Specimens aged for $6 \mathrm{hr}$ or longer at $375^{\circ} \mathrm{F}$ and subsequently exposed without surface protection by anodic treatment for 6 weeks in the marine atmosphere were generally no more severely damaged than the commercially heat-treated material exposed without artificial aging. For one lot of unaged material the effect of stress was marked in increasing the corrosion damage; the ultimate tensile strength of the stressed specimens was reduced 9 percent, as the result of 6 weeks of exposure in the marine atmosphere, compared to 1 percent for the unstressed material; the percent elongations on the two sets of specimens were reduced 53 and 17 percent, respectively. However, there was little difference in the corrosion damage to specimens that had been aged $6 \mathrm{hr}$ or more at $375^{\circ} \mathrm{F}$ whether they were exposed stressed or unstressed in the corroding media.

The maximum losses in tensile strengths and ductilities of unstressed panels, exposed without surface protection to the weather (marine atmosphere for 1 year) or to tidewater (intermittent immersion for 14 days), were found in specimens aged 1 to $2 \frac{1}{2} \mathrm{hr}$ (at $385^{\circ} \mathrm{F}$ ).

5. Intercrystalline corrosion in some degree was found in all of the aged materials on examination after exposure in any of the corroding media. The intercrystalline corrosion was most severe in specimens aged for short periods of time; as the aging periods were increased to or beyond those necessary to produce the maximum values of yield strength, for the various aging temperatures, the intercrystalline corrosion became less general and less severe than that found in specimens aged for the shorter periods. 
The assistance of Houston Babb, Thomas P. Royston, Jr., and Richard C. Rohan; and the cooperation of Willard H. Mutchler and Fred M. Reinhart, of this Bureau; and Lieutenants C. A. Snavely and M. Franklin, of the Naval Air Station, Hampton Roads, Va., in this investigation is gratefully acknowledged.

\section{References}

[1] P. P. Mozley, Elevated temperature aging of $24 \mathrm{~S}$ aluminum alloy, J. Aeronautical Sciences 10, 180 (1943).

[2] J. N. Kotanchik, W. Wood and G. W. Zender, The effect of artificial aging on the tensile properties of Alclad 24S-T and 24S-T aluminum alloys, NACA Bul. RB No. 3H23 (August 1943).

[3] H. L. Logan, Effect of the quenching rate on susceptibility to intercrystalline corrosion of heat-treated 24S aluminum alloy sheet, J. Research NBS 26 , 321 (1941) RP1378.
[4] H. S. Rawdon, Corrosion embrittlement of duralumin, III Effect of the previous treatment of sheet material on the susceptibility to this type of corrosion, NACA Tech. Note 284 (April 1928).

[5] R. B. Mears, R. H. Brown, and E. H. Dix, Jr., A generalized theory of stress corrosion of alloys, p. 329, Symposium on stress corrosion cracking of metals, ASTM and AIME (1944).

[6] Army-Navy Aeronautical Specification, AN-QQ-H$186 \mathrm{a}$, Heat treatment of aluminum alloys, process for, paragraph E-7 (b).

[7] H. S. Rawdon, Corrosion embrittlement of duralumin, V. Results of weather-exposure tests, NACA Tech. Note 304 (February 1929).

[8] Willard Mutchler and W. G. Galvin, Tidewater and weather-expssure tests on metals used in aircraft, NACA Tech. Note 736 (November 1939).

[9] L. E. Simon, Engineers' manual of statistical methods, p. 138 (John Wiley \& Sons, Inc., New York, N. Y., 1941).

Washington, November 22, 1946. 